\title{
The Cassini Campaign observations of the Jupiter aurora by the Ultraviolet Imaging Spectrograph and the Space Telescope Imaging Spectrograph
}

\author{
Joseph M. Ajello ${ }^{\mathrm{a}, *}$, Wayne Pryor ${ }^{\mathrm{b}}$, Larry Esposito ${ }^{\mathrm{c}}$, Ian Stewart ${ }^{\mathrm{c}}$, William McClintock ${ }^{\mathrm{c}}$, \\ Jacques Gustin ${ }^{\mathrm{d}}$, Denis Grodent ${ }^{\mathrm{d}}$, J.-C. Gérard ${ }^{\mathrm{d}}$, John T. Clarke ${ }^{\mathrm{e}}$ \\ a Jet Propulsion Laboratory, California Institute of Technology, Pasadena, CA 91109, USA \\ ${ }^{\mathrm{b}}$ Central Arizona College, Casa Grande, AZ 85228, USA \\ ${ }^{\mathrm{c}}$ Laboratory for Atmospheric and Space Physics, University of Colorado, Boulder, CO 80303, USA \\ ${ }^{\mathrm{d}}$ Laboratoire de Physique Atmosphérique et Planétaire, Université de Liège, 4000 Liège, Belgium \\ e Boston University, Boston, MA 02215, USA
}

Received 13 September 2004; revised 23 January 2005

Available online 9 September 2005

\begin{abstract}
We have analyzed the Cassini Ultraviolet Imaging Spectrometer (UVIS) observations of the Jupiter aurora with an auroral atmosphere two-stream electron transport code. The observations of Jupiter by UVIS took place during the Cassini Campaign. The Cassini Campaign included support spectral and imaging observations by the Hubble Space Telescope (HST) Space Telescope Imaging Spectrograph (STIS). A major result for the UVIS observations was the identification of a large color variation between the far ultraviolet (FUV: 1100-1700 $\AA$ ) and extreme ultraviolet (EUV: $800-1100 \AA$ ) spectral regions. This change probably occurs because of a large variation in the ratio of the soft electron flux (10-3000 eV) responsible for the EUV aurora to the hard electron flux $(\sim 15-22 \mathrm{keV})$ responsible for the FUV aurora. On the

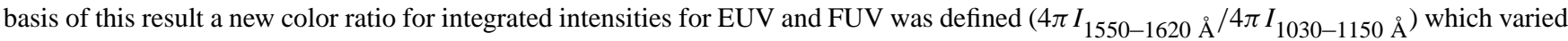
by approximately a factor of 6 . The FUV color ratio $\left(4 \pi I_{1550-1620 \AA} / 4 \pi I_{1230-1300 \AA)}\right.$ was more stable with a variation of less than $50 \%$ for the observations studied. The medium resolution (0.9 $\AA$ FWHM, G140M grating) FUV observations (1295-1345 A and $1495-1540 \AA$ ) by STIS on 13 January 2001, on the other hand, were analyzed by a spectral modeling technique using a recently developed high-spectral resolution model for the electron-excited $\mathrm{H}_{2}$ rotational lines. The STIS FUV data were analyzed with a model that considered the Lyman band spectrum (B $\left.{ }^{1} \Sigma_{\mathrm{u}}^{+} \rightarrow \mathrm{X}^{1} \Sigma_{\mathrm{g}}^{+}\right)$as composed of an allowed direct excitation component $\left(\mathrm{X}^{1} \Sigma_{\mathrm{g}}^{+} \rightarrow \mathrm{B}{ }^{1} \Sigma_{\mathrm{u}}^{+}\right)$and an optically forbidden component $\left(\mathrm{X}^{1} \Sigma_{\mathrm{g}}^{+} \rightarrow \mathrm{EF}, \mathrm{GK}, \mathrm{HH}, \ldots{ }^{1} \Sigma_{\mathrm{g}}^{+}\right.$followed by the cascade transition $\left.{ }^{1} \Sigma_{\mathrm{g}}^{+} \rightarrow \mathrm{B}^{1} \Sigma_{\mathrm{u}}^{+}\right)$. The medium-resolution spectral regions for the Jupiter aurora were carefully chosen to emphasize the cascade component. The ratio of the two components is a direct measurement of the mean secondary electron energy of the aurora. The mean secondary electron energy of the aurora varies between 50 and 200 eV for the polar cap, limb and auroral oval observations. We examine a long time base of Galileo Ultraviolet Spectrometer color ratios from the standard mission (1996-1998) and compare them to Cassini UVIS, HST, and International Ultraviolet Explorer (IUE) observations.
\end{abstract} (C) 2005 Elsevier Inc. All rights reserved.

Keywords: Spectroscopy; Jupiter magnetosphere; Jupiter atmosphere; Ultraviolet observations

\section{Introduction}

Jupiter's UV aurora has the strongest optical signature of

* Corresponding author. Fax: +1 8183549476

E-mail address: jajello@mail.jpl.nasa.gov (J.M. Ajello). the electromagnetic interaction between the magnetosphere and the ionosphere. After the Sun, the jovian aurora is the 
most intense source of UV radiation in the Solar System. Jupiter's aurora deposits far more energy into its upper atmosphere than occurs elsewhere in the Solar System. Its ultimate power source is the rotational energy of Jupiter and plasma processes in the near co-rotating middle magnetosphere. From the time of Voyager in 1979 (Broadfoot et al., 1981) to the present observations by HST STIS and Cassini UVIS in the new millennium (Dols et al., 2000; Ajello et al., 1998, 2001), estimates of deposited power have remained the same for two solar cycles at a level of $\sim 10^{13} \mathrm{~W}$ (requiring an energy input of $\sim 10 \mathrm{erg} / \mathrm{cm}^{2} / \mathrm{s}$ ). Moreover, this enormous power input into the auroral zone presumably controls thermosphere global dynamics (Emerich et al., 1996) and atmospheric chemistry (Perry et al., 1999; Wong et al., 2000). The high spatial resolution capability $(\sim 200 \mathrm{~km})$ of STIS with the two-dimensional multi-anode array MAMA detectors provides FUV images that resolve the polar cap and main auroral oval (Gustin et al., 2002; Grodent et al., 2003a, 2003b).

The modeling of the dynamical magnetosphere-ionosphere coupling causing the jovian aurora has been aptly described by Hill (2001), Bunce and Cowley (2001), and Cowley and Bunce (2001). These authors suggest that the auroral oval indicates the presence of a global-scale Birkeland current system that maps to $\sim 30 R_{\mathrm{j}}$. This current system passes planetary angular momentum to the outward moving plasma sheet maintaining the middle magnetosphere in near co-rotation (Hill, 2001). In the region of upward currents (downward electrons), field-aligned potentials accelerate electrons to auroral energies $(10-100 \mathrm{keV}$ ) (Mauk et al., 2003).

The flyby of the Cassini spacecraft past the jovian planetary system allowed a long observation period (approximately October 1, 2000 to March 22, 2001) dubbed the Cassini Campaign (CC). The remote sensing instruments taking part in the CC auroral observations were UVIS, STIS, Far Ultraviolet Spectrometer Explorer (FUSE), Chandra and Infrared Telescope Facility (IRTF). In this paper, along with the companion paper by Pryor et al. (2005), we describe the CC auroral observations by UVIS. Herein we model the closest approach aurora spectra that were obtained in the period from December 29, 2000 (DOY 364) to January 2, 2001 (DOY 2) with the spacecraft distance changing from 138 to $142 R_{\mathrm{j}}$. The UVIS instrument provided complete $\mathrm{H}_{2} \mathrm{Ry}-$ dberg band spectra coverage from 800 to $1700 \AA$ at both 2.4 and $4.8 \AA$ FWHM. The UVIS is the second Jupiterobserving instrument to achieve this spectral resolution over the full spectral range of Rydberg bands after the Hopkins Ultraviolet Spectrograph (HUT), which measured a single spectrum of the jovian aurora in November 1995 at $3.0 \AA$ FWHM. The UVIS data archive is much more extensive consisting of tens of spectra of the aurora. The temporal variations of the complete UV spectrum during the flyby are particularly striking. The variation is interpreted in terms of temporal variations of the ratio of the soft electron component that excites the EUV (Ajello et al., 2001) to the hard electron flux that excites the FUV (Ajello et al., 1998). Additionally, we study spatially resolved medium resolution spectra (0.9 A FWHM with the G140M grating) from STIS obtained during the CC on January 13, 2001. A recently developed high-resolution code (Liu et al., 2002) for modeling the intensity of the $\mathrm{H}_{2}$ rotational lines from the STIS spectra allows a different approach to the modeling of the Lyman band spectrum by providing very accurate computations of the separate contributions to the spectra from direct excitation and cascading. The contribution of cascade to the measured outgoing portion of the FUV spectrum is predominantly excited by low-energy secondary electrons (Ajello et al., 1998). The cascade cross-section from the EF, GK, and $\mathrm{H} \overline{\mathrm{H}}$ states of $\mathrm{H}_{2}$ is optically forbidden with a cross-section that is sharply peaked at low electron energy $(\sim 20 \mathrm{eV})$ (Liu et al., 2003). STIS also acquired complete FUV spectra with the G140L grating; one of these observations on December 28 occurred close in time to the December 29 closest approach UVIS measurement. The spectra from the two spacecraft are analyzed independently, since each spacecraft observed different aurora.

\section{The observations}

The observations for the Jupiter aurora by UVIS during the CC began in October 2000. There were approximately $100 \mathrm{~h}$ of viewing the aurora and about 100 observations of various kinds (torus, satellite and Jupiter disk) by the time the CC ended on March 22, 2001. Some Jupiter system observations of the disk had the slit-oriented parallel to the jovian equator to simultaneously measure and spatially resolve the aurora and torus emissions during far encounter. Later observations had the slit oriented perpendicular to the jovian equator, an effective way to simultaneously observe the north and south aurora on separate spatial pixels of the imaging detector. To achieve observations that separately resolve the north and south auroral regions required a narrow time window around closest approach of the Cassini flyby. The UVIS could achieve a minimum of eight-pixel spatial resolution of the Jupiter disk for the period of \pm 15 days around closest approach. Closest approach occurred on December 30, 2000 with a disk diameter of $14 \mathrm{mrad}$.

The characteristics of the UVIS instrument have been discussed in detail in a recent paper (Esposito et al., 2004). In brief, the instrument consists of separate telescopes for the EUV (563-1182 ̊) and FUV (1115-1913 $\AA$ ) channels, respectively, with an option of one of three separate entrance slits for each instrument: low resolution $(75,100 \mu \mathrm{m}$ [FUV, EUV] slit widths), high resolution $(150,200 \mu \mathrm{m}$ [FUV, EUV] slit widths) and occultation slit $(800,800 \mu \mathrm{m}$ [FUV, EUV] slit widths). Each configuration determines the field-of-view (FOV) in the plane of dispersion. The UVIS field-of-view is $1 \mathrm{mrad}(206 \mathrm{arcsec}) \times 59 \mathrm{mrad}$ for the EUV high-resolution channel and $0.75 \times 60 \mathrm{mrad}$ for the FUV high-resolution channel in the directions of the slit width 
Table 1

Summary of the UVIS aurora observations

\begin{tabular}{|c|c|c|c|c|c|c|}
\hline Date & $\begin{array}{l}\text { Time at start } \\
\text { (GMT) }\end{array}$ & $\begin{array}{l}\text { Duration } \\
(\mathrm{min})\end{array}$ & $\begin{array}{l}\text { Distance at start } \\
\left(R_{\mathrm{j}}\right)\end{array}$ & $\begin{array}{l}\text { CML at start of } \\
\text { observation }\end{array}$ & Slit & Pole \\
\hline 29 December 2000 & 02:20 & 33.3 & 138 & $285^{\circ} \mathrm{W}$ & Low & North \\
\hline 29 December 2000 & $02: 20$ & 33.3 & 138 & $285^{\circ} \mathrm{W}$ & Low & South \\
\hline 02 January 2001 & 06:03 & 277.3 & 142 & $290^{\circ} \mathrm{W}$ & High & South \\
\hline
\end{tabular}

Table 2

HST observations during Jupiter Millennium Campaign

\begin{tabular}{llllllll}
\hline Date & $\begin{array}{l}\text { Start time } \\
\text { (UT) }\end{array}$ & $\begin{array}{l}\text { Exposure } \\
\text { (s) }\end{array}$ & Grating mode & $\begin{array}{l}\text { Slit } \\
\left(\operatorname{arcsec}^{2}\right)\end{array}$ & $\begin{array}{l}\text { Bandwidth } \\
(\AA)\end{array}$ & $\begin{array}{l}\text { Central wavelength } \\
(\AA)\end{array}$ & $\begin{array}{l}\text { North/ } \\
\text { south }\end{array}$ \\
\hline 28 December 2000 & $07: 15: 22$ & 630 & G140L & $52 \times 0.5$ & 590 & 1425 & 1425 \\
28 December 2000 & $07: 31: 22$ & 630 & G140L & $52 \times 0.5$ & 590 & South \\
13 January 2001 & $16: 58: 07$ & 480 & G140M & $52 \times 0.5$ & 56 & 1321 & South \\
13 January 2001 & $17: 13: 50$ & 480 & G140M & $52 \times 0.5$ & 56 & 1518 & North \\
\hline
\end{tabular}

and height, respectively. The FOV widths double to 2.0 and $1.5 \mathrm{mrad}$, respectively, for the low-resolution measurements of the EUV and FUV channels. The data discussed in this paper are for the low and high-resolution slits for each of the EUV and FUV channels. The detector is a Codacon (CODed Anode array CONverter). The detector format is $1024 \times 64$ (spectral $\times$ spatial) pixel array with a pixel size of $25 \times 100 \mu \mathrm{m}$. Thus in the high-resolution mode a monochromatic line will be imaged on 4 pixels FWHM in the EUV and 3 pixels FWHM in the FUV. The instrument slit function is triangular with extended wings. The photocathode materials are $\mathrm{KBr}$ for the EUV and CsI for the FUV. The peak sensitivities occur at $900 \AA$ for the EUV and $1300 \AA$ for the FUV. The instrument operates in the spectral mode by summing rows of the array detector and in the spatial mode by summing columns of the array detector.

The Cassini observations to be discussed in this paper occurred on December 29, 2000 (29Dec00) and January 2, 2001 (02Jan01). We summarize the geometry for these three observations in Table 1 . The observations, 29Dec00 and 02Jan01 were able to resolve the north and south aurora. The three auroral observations listed in Table 1 were very strong providing high $\mathrm{S} / \mathrm{N}$ (signal/noise). The aurora for the north auroral zone on 02Jan01 was very weak and is not listed.

The two HST STIS observations performed during the $\mathrm{CC}$ are described spectra in Table 2 . The primary data set consists of STIS low-resolution spectra $(\Delta \lambda=12 \AA$ FWHM grating G140L, the wavelength band-pass from 1120 to $1736 \AA)$ and medium-resolution spectra $(\Delta \lambda=$ $0.9 \AA$ FWHM, grating G140M, the wavelength band-pass of close to $50 \AA$ centered at 1321 and $1518 \AA$ ). The data sets analyzed in this paper are the G140L observations from 28 December 2000 (28Dec00) and G140M observations of 13 January 2001 (13Jan01). The Jupiter auroral spectra at low resolution with the G140L grating cover the entire FUV (1150-1700 ̊). The $50 \AA$ wavelength spans for the G140M spectral ranges were carefully chosen to include one short wavelength range (1295-1345 $\AA$ ), where the $v^{\prime}=0$ vibrational sequence member $(0,4)$ of the cascade-driven Ly- man bands (B-X) is especially strong and lies near $1335 \AA$ (Dziczek et al., 2000; Liu et al., 2002). The optically forbidden excitation transition $\mathrm{X}^{1} \Sigma_{\mathrm{g}}^{+} \rightarrow \mathrm{EF}, \mathrm{GK}, \mathrm{HH}, \ldots{ }^{1} \Sigma_{\mathrm{g}}^{+}$ from the ground state to the gerade Rydberg series is followed by a cascade transition, $\mathrm{EF}, \mathrm{GK}, \mathrm{HH}, \ldots{ }^{1} \Sigma_{\mathrm{g}}^{+} \rightarrow \mathrm{B}$ ${ }^{1} \Sigma_{\mathrm{u}}^{+}$. We refer to the collection of double minima gerade states EF, GK, $\mathrm{HH}, \ldots$ as EF. The nomenclature of gerade (g) (even) and ungerade (u) (odd) is a center of symmetry reflection property of electronic states of a homonuclear molecule. The electric dipole selection rule behavior for electronic transitions of homonuclear molecules, described in Herzberg (1950), requires that $\mathrm{g} \rightarrow \mathrm{u}$, e.g., the Lyman and Werner band systems.

The longer wavelength range of HST STIS G140 M covers the range from 1495 to $1545 \AA$, where the methane absorption is weak and acetylene absorption strong near $1520 \AA$. Cascade is also weaker in this spectral region (Liu et al., 2002). The spectral image observations were separated by 15 min with start times of 16:58 GMT for the short wavelength spectrum and 17:13 GMT for the long wavelength spectrum. The duration of each spectrum is $6 \mathrm{~min}$. Since the timescale of Jupiter auroral changes in the polar cap region can be minutes (Pryor et al., 2001; Grodent et al., 2003a; Gérard et al., 2003) and the CML (central median longitude) was different in each case, it is not reasonable to think of these spectra as simultaneous. The CMLs for the first spectrum were $223.4^{\circ}$ and $228.3^{\circ}$ at the start and at the end of the exposure, respectively. For the second spectrum, the CMLs were $232.9^{\circ}$ and $237.8^{\circ}$, respectively.

The entrance slit length and width determine the FOV, which was $52 \times 0.5 \operatorname{arcsec}^{2}$ for the G140M observations in the spectral mode. In parallel with the spectral observations in the TIMETAG mode, there were near-simultaneous images from STIS to provide the morphology of the Jupiter auroral zone (aurora oval, polar cap, Io foot). A previous set of STIS data from the Jupiter aurora in 1999 was obtained with the G140M grating (Gustin et al., 2002). We show in Fig. 1A a STIS image of the northern aurora during the CC 


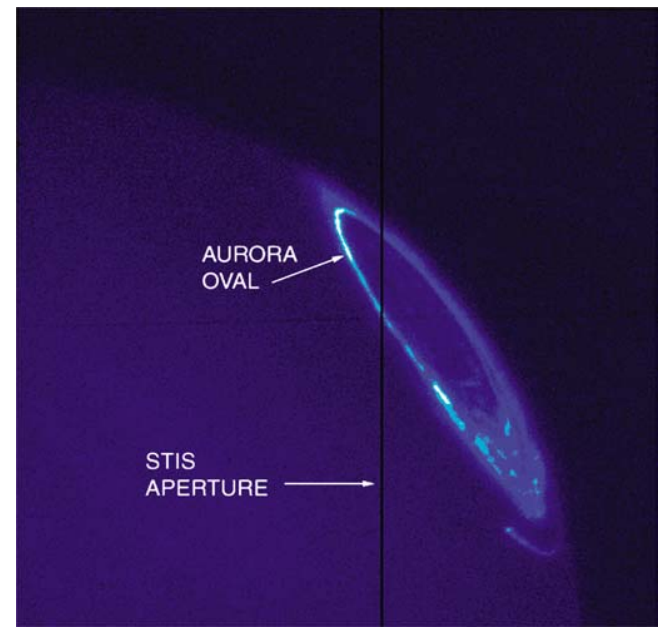

(A)

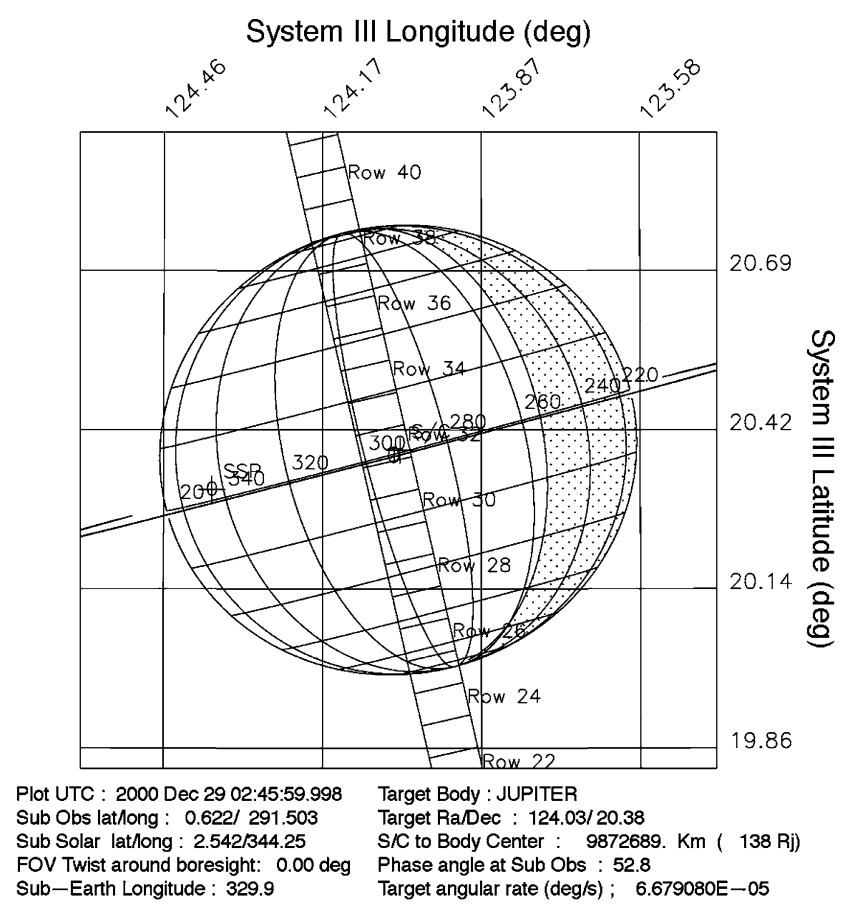

(B)

Fig. 1. (A) A Jupiter STIS image taken at 16:50 UT on 13Jan01 showing the 3 auroral zones of polar cap, auroral oval and limb with the $52 \times 0.5 \operatorname{arcsec}^{2}$ slit projected on the image. The image was taken with a $\mathrm{SrF}_{2}$ filter and the FUV MAMA detector. The MAMA detector has a FOV of $25^{\prime \prime} \times 25^{\prime \prime}$ with an angular resolution of $0.024^{\prime \prime} /$ pixel. (B) The geometry of the UVIS observation for 29Dec00 showing the low-resolution FUV spectrometer slit projected on to the Jupiter disk. The rows numbered 0-60 represent the spatial pixels of the Codacon detector $(1024 \times 64)$. The spacecraft was 138 $R_{\mathrm{j}}$ from the planet.

taken 8 min before the G140M spectra. The black line is the approximate image location of the slit at 16:58 UT.

\section{The Cassini auroral analysis}

The Cassini analysis can be divided into two subsections. The data reduction of the raw data packets to produce cal- ibrated spectra of the aurora requires proper subtraction of the background. The background subtraction takes place in several steps to be described in the next subsection. The auroral science analysis with the two-stream electron transport code coupled to the spectral code will be described in the subsequent subsection.

\subsection{Data reduction}

In the analysis we concentrate on Jupiter auroral observations acquired at closest approach. These observations occurred on 29Dec00 and 02Jan01. The geometry for the lowresolution FUV slit for the 29Dec00 observation is shown in Fig. 1B and can be compared to the higher resolution HST STIS FOV from Earth-orbit on 13Jan01 shown in Fig. 1A. The locations of the 64 spatial pixels of the Codacon detector are also indicated. The boresights for the EUV and FUV slits are offset by $1 \mathrm{mrad}$ in azimuth (plane of dispersion). The small angular pointing difference is averaged over the data records during the long durations of the observations, generally $1 / 2 \mathrm{~h}$ or more. There is a $50 \%(0 \%)$ overlap in the fields of view of the low-resolution slits (high-resolution slits). At a spacecraft-Jupiter distance of $138 R_{\mathrm{j}}$ the observation points of the EUV and FUV on the planet differ by $10,000 \mathrm{~km}$. The planet must rotate $8^{\circ}(\sim 13 \mathrm{~min})$ to allow the second channel to achieve the same boresight pointing direction to the aurora as the first channel. The geometry plot in Fig. 1B is shown at roughly the center point of the 29Dec00 observation sequence. The UVIS maintained constant boresight pointing at a fixed right ascension and declination as the planet rotated beneath the boresight. In both UVIS observations the EUV and FUV detector spatial pixels numbered from 26 to 40 were on the planet with the smaller number associated with the south pole. Prangé et al. (1998) have shown that in the absence of auroral storms or localized bright spots that the longitudinal characteristics of the Jupiter aurora are smoothly varying with medium scale brightness variations extending over $5^{\circ}-20^{\circ}$ in longitude. An extended time period study of the aurora for seven days during the $\mathrm{CC}$ from the HST observations listed in Table 2 by Grodent et al. (2003b) has shown that the main oval is stable for periods of tens of minutes to hours. We examine auroral data from one row (or two rows, if the aurora is on the edge of two pixels) of the UVIS spatial pixel(s) for each pole. The signal from these one or two rows may contain a contribution from the polar cap aurora. In this paper, for the purposes of analysis of the extended time durations (30 min or more) of the aurora we assume the two-channel boresights are co-aligned.

The observational data is acquired as an image on the detector. The data is read out in individual records that are equally spaced in time; and the records are summed to present a unified observational spectrum of high S/N observation. Each record can have a duration of approximately 1-30 min. The record length for the 29Dec00 observation is $1000 \mathrm{~s}$ with a total of two records of auroral data. The 3D (dimensional) detector image of the slit in both spatial 


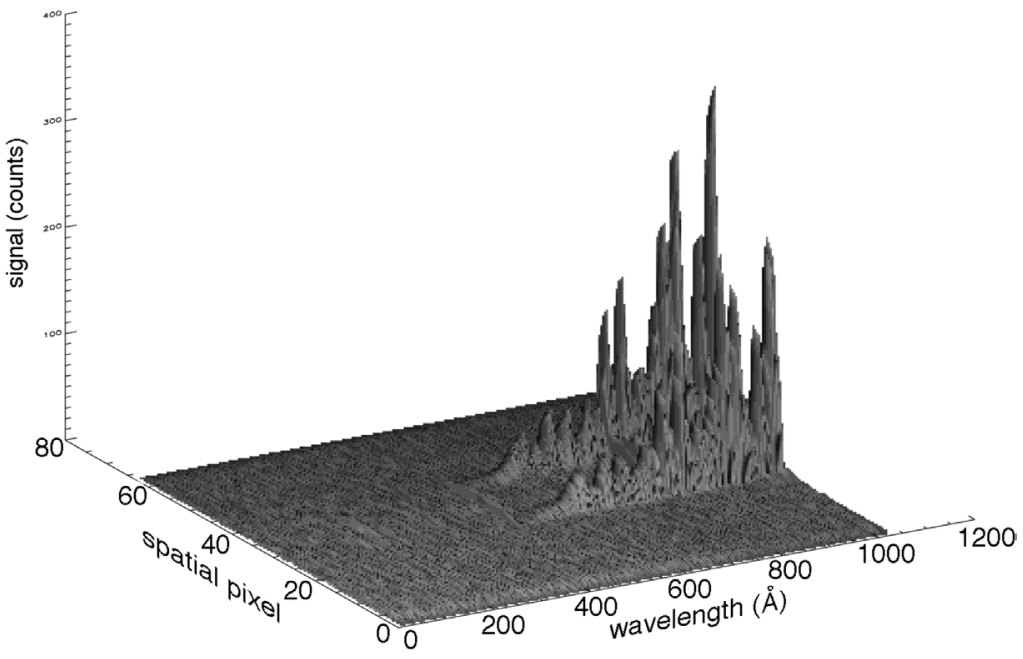

(A)

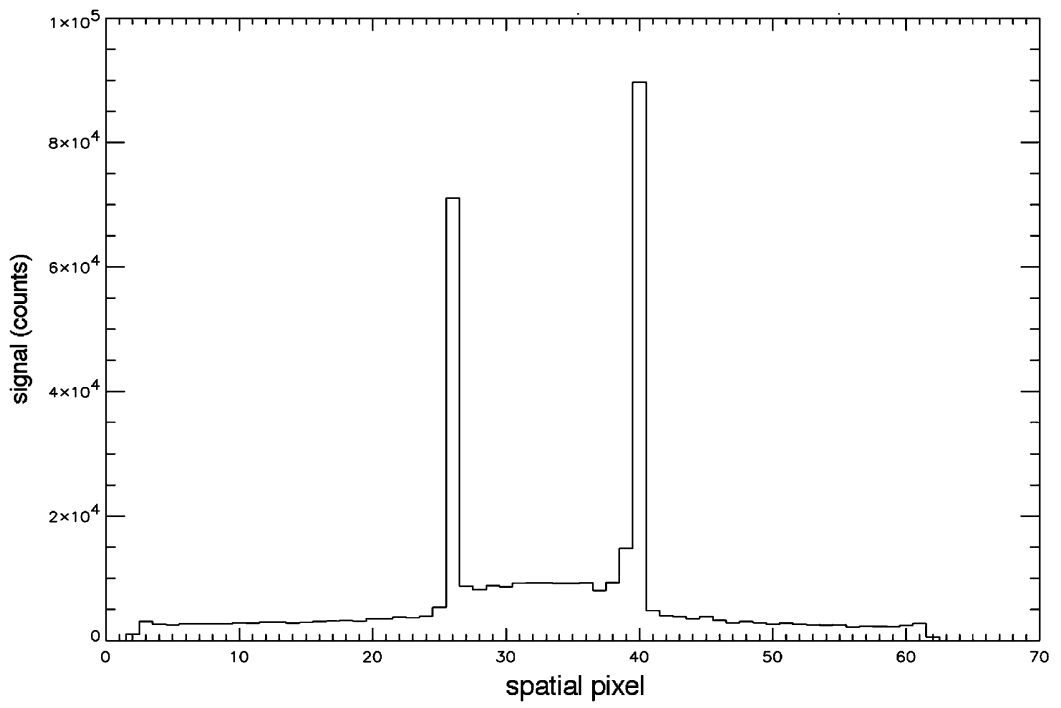

(B)

Fig. 2. (A) A three-dimensional surface plot of the EUV channel signal in counts plotted vs wavelength and spatial pixel. The EUV spectral-spatial image of the jovian system clearly demarks an active UV auroral oval in both the northern and the southern polar regions for 02:20 29Dec00 observation of 33.3 min. (B) The total number of EUV channel counts in each spatial pixel summed over all spectral channels for the $29 \mathrm{Dec} 00$. The peaks in rows 26 and 40 correspond to the locations of the south and north auroral oval, respectively as shown in Fig. $1 \mathrm{~B}$.

and wavelength pixels for 29Dec00 is shown in Fig. 2A. The figure indicates strong aurora in both the north and south from spatial pixels associated with the poles. The presence of the aurora is indicated by the strong $\mathrm{H}_{2}$ Rydberg bands from 850 to $1200 \AA$, lying upon one or two spatial rows. The auroras are found on either side of the low latitude dayglow. The dayglow is observed since the solar phase angle, which is listed in Fig. 1B, is less than $90^{\circ}$ for the auroral sequence. A similar 3D plot can be made for the 02Jan01 data except that the recorded data showed only one strong aurora in the south. The next step is to carefully identify and separate the pixels containing the aurora as opposed to the weak dayglow. The specific rows of pixels that contain the aurora are obtained by summing the 3D data over wavelength (all columns) and is shown in Fig. 2B for 29Dec00. We find the aurora in one or two rows for each of the two auroral zones on 29Dec00. The northern aurora is on spatial pixel(s) (40 for the EUV and 38, 39 for the FUV) and the southern aurora is on spatial pixel 26 for the EUV and 24, 25 for the FUV. Similarly for the south on 02Jan01 the southern aurora lies on rows 7 and 8 and a weak northern aurora in rows 20 and 21 for both the EUV and FUV. To this pixel number for 02Jan01 must be added the windowing offset of 20 rows, since only 24 rows (numbered 20-43) were readout for transmission to the ground for the 02Jan01 data. Thus, the southern aurora was observed on spatial pixels 26 and 27 (referenced to a first spatial pixel of number 0). The locations of the aurora as determined from the FUV channel for both sets of observations were the same as for the EUV channel.

We will describe in detail the continuing data analysis preparation of the $02 \mathrm{Jan} 01$ southern aurora, since the 
02Jan01 data contains only one strong aurora. Our first step is to arrange the data in the spectral mode for the south aurora rows $(7,8)$ and for the north aurora $(20,21)$. The dayglow in rows (9-19) will be described in a separate paper. The auroral oval has been observed at high spatial resolution by the Galileo Solid State Imaging Subsystem (SSI). The SSI showed that the oval width varies between 200 and $1000 \mathrm{~km}$ (Ingersoll et al., 1998; Vasavada et al., 1999). The UVIS FOV of a pixel projects to a width of about $10^{4} \mathrm{~km}$ (compared to $200 \mathrm{~km}$ for HST STIS) at a distance of $142 R_{\mathrm{j}}$ from the planet for this distant flyby. For the 02Jan01 observation it is likely the north and south aurora were observed near the edge of a pixel and spanned two pixels. A fill-factor (Ajello et al., 1998) of 0.1 is arbitrarily assumed throughout this paper for the closest approach aurora observations and corresponds to an auroral main oval width of $1000 \mathrm{~km}$. The Cassini UVIS does not have sufficient spatial resolution to study the structure of the auroral oval by itself and may include some contribution from the polar cap. With the added uncertainty of polar cap auroral contributions to the signal (Grodent et al., 2003a, 2003b) the absolute auroral brightness and energy input calculations deduced in the modeling are approximate. The period of observation of the 02Jan01 aurora was extensive. The summed signal in counts is a co-addition of individual spectra for nearly half a jovian rotation period (Table 1). The 29Dec00 auroral data archive was reduced by the same method and its composite integration time is $33.3 \mathrm{~min}$. The $02 \mathrm{Jan} 01$ data were obtained in 177 consecutive records of $94 \mathrm{~s}$ each for a total duration of $277.3 \mathrm{~min}$. The measured and unreduced signal consists of composite contributions from the aurora and foreground UV emissions from the interplanetary Lyman- $\alpha$ as well as instrumental and spacecraft Radioisotope Thermal Generator (RTG) background signals.

The next step is to remove these spurious signals and to produce a raw auroral spectrum prior to calibration. We need to subtract from the total signal foreground signal the contributions from the torus emissions, background noise from random detector noise and RTG background, foreground scattered signal from interplanetary $\mathrm{H}$ Ly- $\alpha$ plus the disk $\mathrm{H} \mathrm{Ly}-\alpha$, producing internally scattered light by the grating. There is an additional source of internally scattered light from a pinhole light leakage (which the UVIS Team refers to as the mesa from its spectral signature) in the EUV channel. First, we can estimate the constant background from RTG plus detector noise background from noise signal data on the first few pixels of the auroral spectrum since the wavelengths near $562 \AA$ do not include any wavelength dependent background effects.

We describe the three main wavelength dependent background contributions to the total signal. The torus foreground, the internally scattered light from interplanetary $\mathrm{H}$ Ly- $\alpha$ /disk backgrounds and the mesa are wavelength dependent backgrounds. The torus signal present as a contamination in all auroral spectra is very strong at OII, III $833 \AA$ and allows us to normalize the auroral + torus signal to a pure torus signal acquired nearby in time for torus signal subtraction. The torus background was acquired independently on 5Dec00 and was normalized to the 02Jan01 torus emission of the OII, III feature at $833 \AA$.

The mesa spectrum of scattered light from light leakage of stellar sources and Jupiter is present on all spectral rows. We employ spectral rows 0-3 (see Fig. 2A) which are viewing into space as the basis for subtraction of the mesa signal contribution to the auroral spectrum.

The one remaining wavelength dependent background signal for the wavelengths above $1150 \AA$ arises from internal instrument scattering from $\mathrm{H} \mathrm{Ly}-\alpha$. Its effect on the auroral data is accounted for by normalizing to the interplanetary $\mathrm{H}$ Ly- $\alpha$ signal in the EUV channels as measured during cruise. The spectral range of the 1024 channels of the EUV channel stops at $1182 \AA$ and does not include H Ly- $\alpha$. The contribution of the H Ly- $\alpha$ blue wing to the EUV signal is small below $1170 \AA$. The contribution of the interplanetary H Ly- $\alpha$ to the raw spectrum is estimated to be less than $7 \%$ at $1150 \AA$ based on comparison to an $\mathrm{H}_{2}$ model in the 1100-1200 $\AA$ range. The Jupiter auroral raw spectrum is formed from the subtraction of all noise sources discussed above. The calibrated spectrum of the EUV channel is shown in Fig. 3A.

We must combine the EUV spectrum with the FUV spectrum. We perform a similar data reduction process for the FUV observation of 02Jan01. The raw FUV spectrum shows the effect of the Rayleigh scattered solar reflectance by Jupiter longward of $1700 \AA$ during the daylight observation. Three-point smoothing is needed to partially eliminate the fixed pattern pixel-pixel noise from the FUV channel Codacon. The calibrated spectrum of the FUV channel is shown in Fig. 3B. The FUV spectrum is dominated by the Lyman bands, Werner bands and Lyman- $\alpha$ attenuated by overlying hydrocarbons.

The wavelength overlap by the EUV with the FUV is small. The FUV spectral range extends from 1115 to $1913 \AA$. Fig. 3A shows that the EUV spectrum extends from 563 to $1182 \AA$. However, the sensitivity of the FUV channel is not reliable below $1150 \AA$, based upon the sharp cutoff characteristics of the transmission curve of the $\mathrm{MgF}_{2}$ window. Thus the region of the calibrated EUV spectrum above $1150 \AA$ is particularly important in properly overlapping the EUV and FUV spectra. The overlap region will be discussed in detail later.

\subsection{The analysis}

The analysis made use of the two-stream electron transport code that includes thermal transport to determine the thermal balance and altitude variation of Jupiter's auroral atmosphere (Grodent et al., 2001). Recent improvements to the best-estimate of the functional form of the incident primary electron flux (Grodent et al., 2001) include a mixture of three energy distributions: (1) a high-energy component $15 \mathrm{keV}$ (diffuse aurora) to $22 \mathrm{keV}$ (discrete aurora) determined by the measured FUV color ratio (Yung et al., 


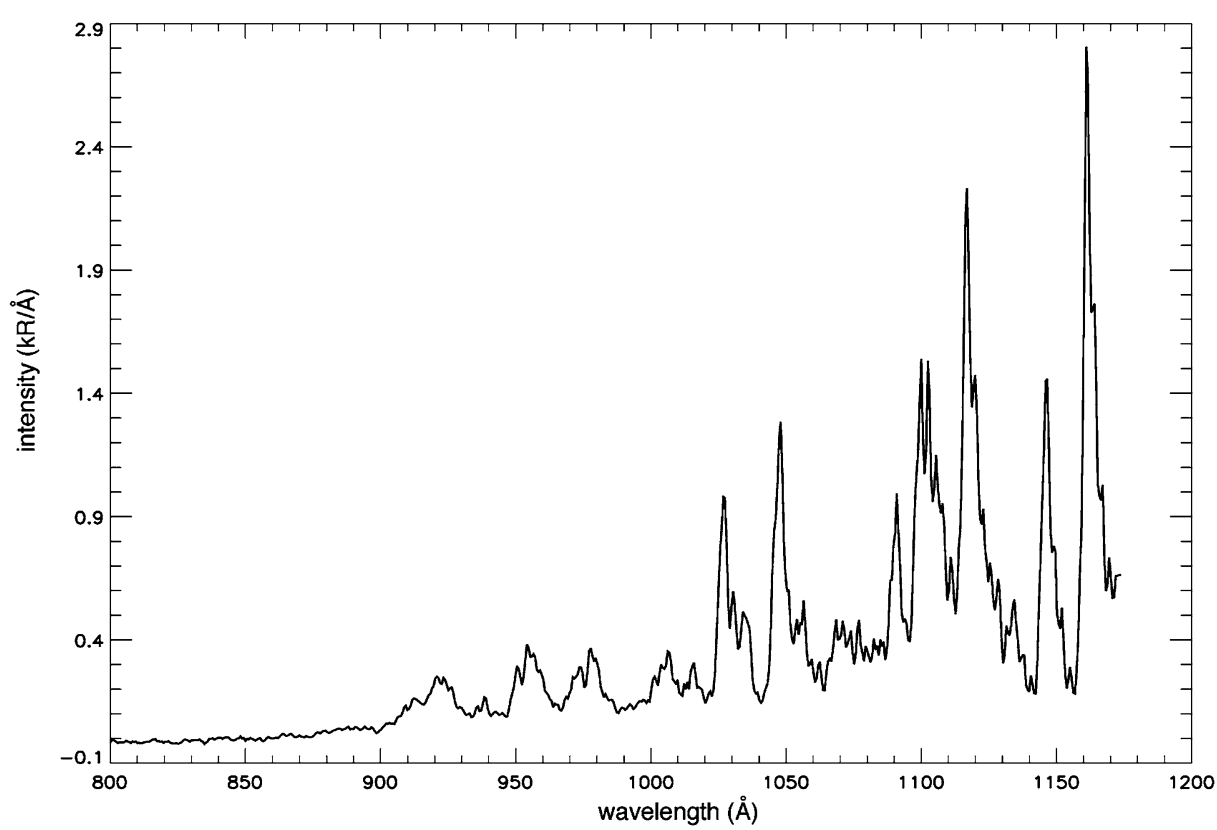

(A)

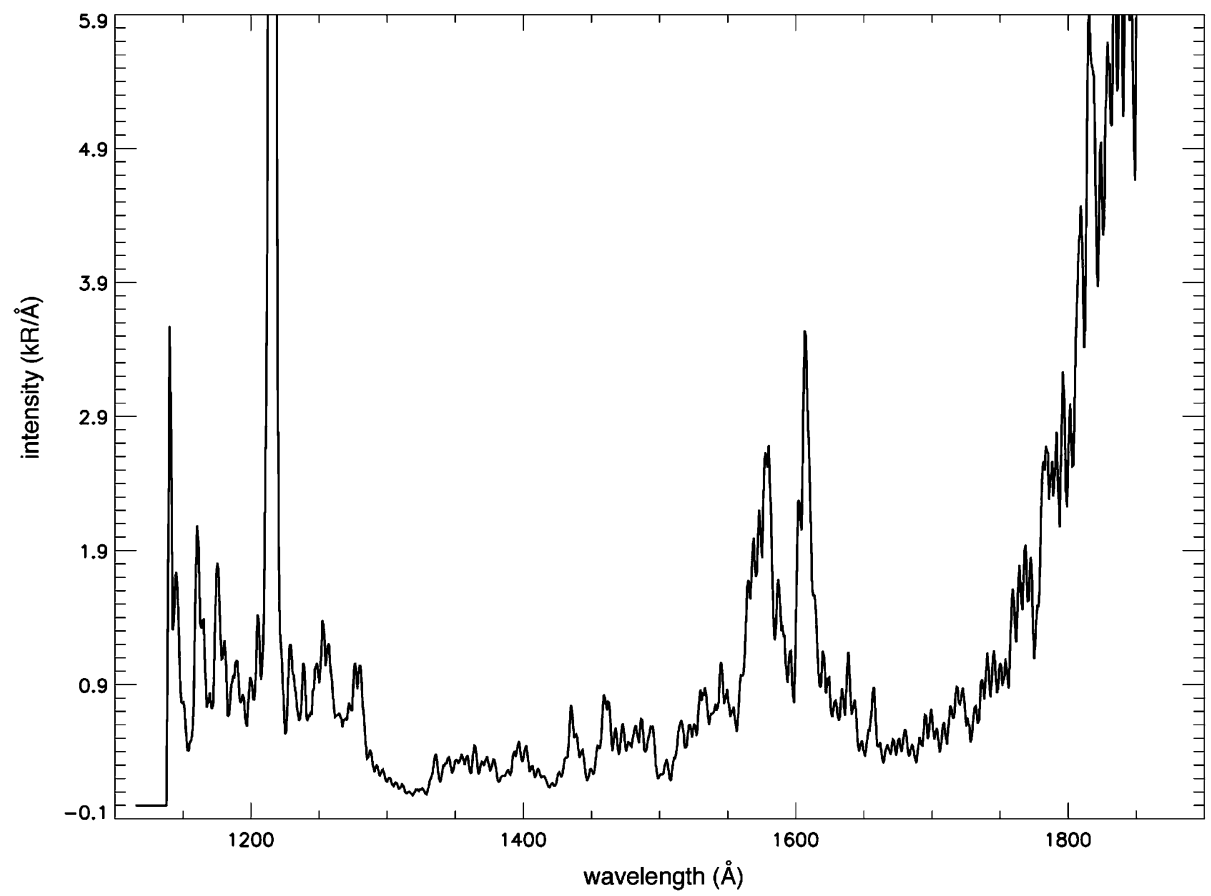

(B)

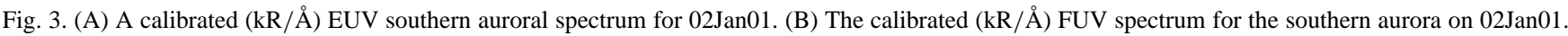
The data was smoothed by a 3-channel box-car smoothing function. Each channel is $0.8 \AA$ wide.

1982) (the high-energy component heats the homosphere), (2) a soft electron component near $3 \mathrm{keV}$ for heating the thermosphere, and (3) a weak $100 \mathrm{eV}$ component for heating the upper thermosphere and controlling the exobase temperature.

We use the same approach to modeling the UVIS calibrated and merged spectra as Ajello et al. (2001) has prescribed for the analysis of Galileo and HUT spectra. The new two-stream model employed here more accurately describes the low-energy primary electron flux distribution function from 10 to $3000 \mathrm{eV}$ (Grodent et al., 2001) and is based on the known thermal constraints of the auroral atmosphere. Our model is a synthesis of the following: (1) the spectral line $\mathrm{H}_{2}$ Rydberg code for electron impact described by Ajello et al. (1984, 1998) and Shemansky and Ajello (1983), (2) the two-stream electron energy degradation transport code derived by Grodent et al. (2001), and (3) the one-dimensional heat conduction equation which includes the heating of the atmosphere by the auroral electron precipitation (Grodent et al., 2001). 


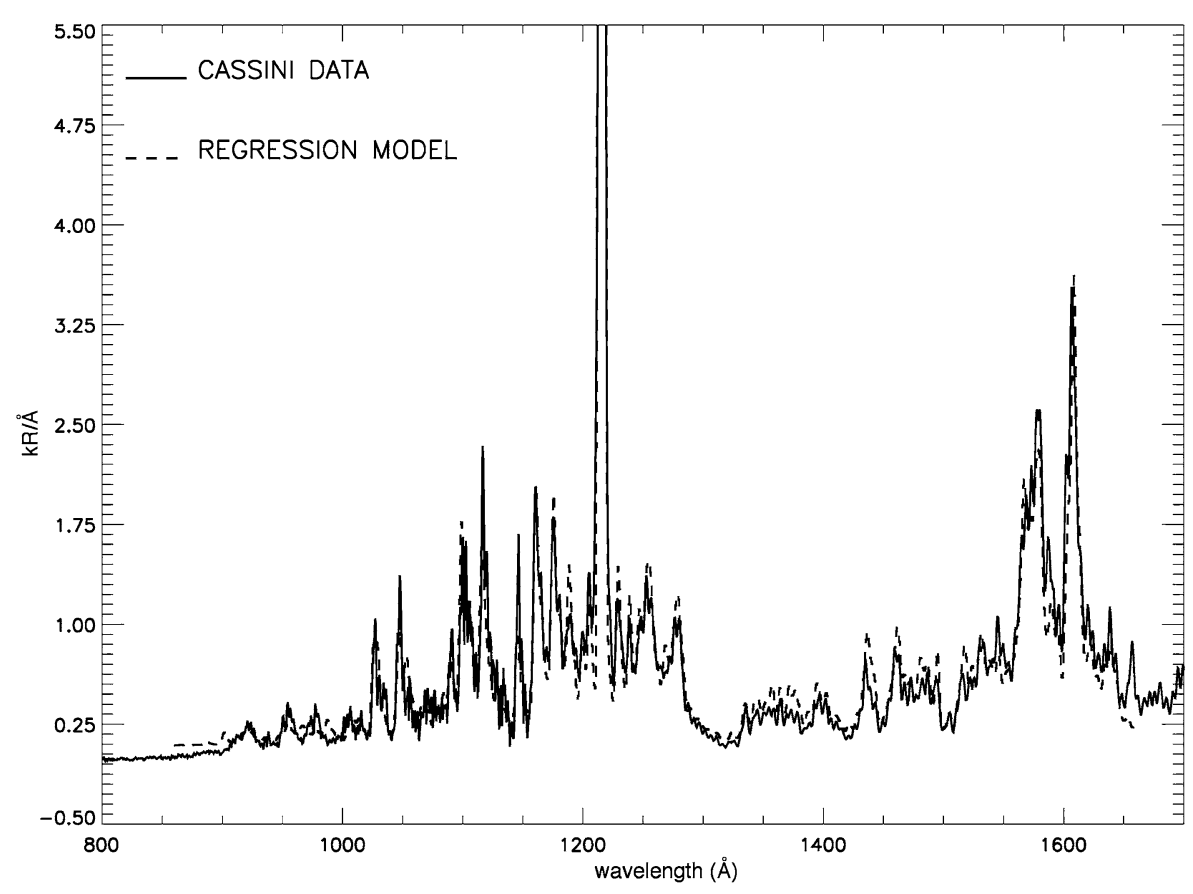

Fig. 4. The Cassini composite spectrum in units of $\mathrm{kR} / \AA$ for the southern aurora of 02Jan01 fitted in linear regression analysis with: (1) a discrete aurora electron flux (Grodent et al., 2001) incident upon an auroral atmosphere with an altitude dependent secondary electron distribution and atmospheric distribution from a two-stream code, and (2) a H Ly- $\alpha$ line. The EUV (0.6 ̊ per channel) and FUV (0.8 A per channel) data are smoothed by a 5-channel box-car smooth prior to merging. The model (2.4 А FWHM with $0.6 \AA$ channels) is smoothed by a 5-channel box-car smooth.

The atmosphere is divided into 200 layers of vertical depth 10-20 km from 0 to $4000 \mathrm{~km}$ (Ajello et al., 2001). The emission emerging at the top of the atmosphere from every layer is added to the running sum from all previous layers until the top of the atmosphere is obtained. Model line spectra are generated for molecular hydrogen for the thermodynamic conditions at each layer. For each layer a model line spectrum is produced from each differential electron flux element in the secondary electron spectrum from the threshold for the Lyman bands at $11 \mathrm{eV}$ to very high electron impact energy $(\sim 500 \mathrm{keV})$. These 70 energy-dependent spectra are summed to give the emission from each layer. Self-absorption and hydrocarbon absorption from the actual heated auroral atmosphere determine the attenuation in the UV spectrum from each layer. The emerging line spectrum is convolved with the instrument function (Esposito et al., 2004). The model is compared with the spectral observations to determine for the 29Dec00 and 02Jan01 observations the energy input, $Q_{0}$, to the aurora. The EUV channel alone can not determine $E_{0}$, the characteristic energy of the auroral flux. The EUV spectrum is produced high in the auroral atmosphere above the main deposition peak. The spectral distribution is sensitive to the overlying $\mathrm{H}_{2}$ column by self-absorption processes. The FUV spectrum has little absorption from methane above wavelengths of $1400 \AA$ or self-absorption from $\mathrm{H}_{2}$ above wavelengths of $1200 \AA$. The long wavelengths of the FUV channel have a preponderance of signal from deep in the homosphere (200-300 km above the 1 bar level). The FUV color ratio determines $E_{0}$ (Dols et al., 2000).
We compare the data and model using a regression analysis best-fit approach. The regression model, based on our knowledge of the source of the aurora emission, is composed of two vectors-one vector is composed of the $\mathrm{H}_{2}$ Rydberg bands and the second vector is the H Ly- $\alpha$ line. We show in Fig. 4 the best-fit regression model to the 02Jan01 south observation. The model no longer needs the ad hoc $20 \mathrm{eV}$ laboratory spectrum as was used in the analysis of the Galileo and HUT observations (Ajello et al., 2001). The Grodent et al. model already includes the very low-energy primary electrons $(\sim 100 \mathrm{eV})$. The brightness of the Rydberg bands (without the $\mathrm{H} \mathrm{Ly}-\alpha$ line) emerging from the atmosphere is $496 \mathrm{kR}$ as measured by the Cassini UVIS assuming a fill factor of 0.1 and the cosine of the emission angle is 0.3 (emission angle of $72.5^{\circ}$ ). The energy input into the atmosphere required to maintain this intensity is $74 \mathrm{erg} / \mathrm{cm}^{2} / \mathrm{s}$. There is approximately a $10 \%$ efficiency of conversion of auroral primary electron energy input to the UV emission in the Rydberg bands (Waite et al., 1983).

We show in Figs. 5A and 5B the best-fit regression models to the 29Dec00 north observation. The brightness of the Rydberg bands (without the $\mathrm{H} \mathrm{Ly}-\alpha$ line) is $550 \mathrm{kR}$ as measured by the Cassini UVIS. The energy input into the atmosphere required to maintain this intensity is $96 \mathrm{erg} / \mathrm{cm}^{2} / \mathrm{s}$.

The main discrepancy between the model and data occurs in the neighborhood of the strong EF bands. The recent modern spectral model of the EF bands developed by Liu et al. (2002) to be used in the next section has not yet been incorporated into the two-stream spectral model used here. 

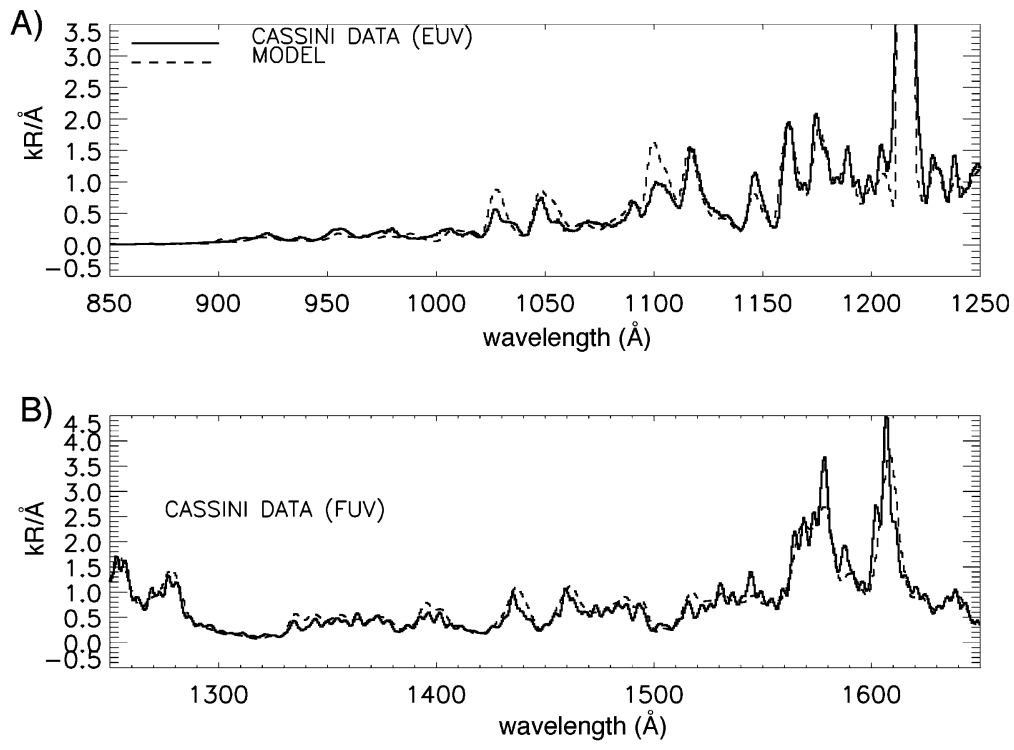

Fig. 5. (A) The Cassini EUV wavelength region of the UV spectrum in units of kR/A for the southern aurora of 29Dec00 fitted in linear regression analysis with: (1) a discrete aurora electron flux (Grodent et al., 2001) incident upon an auroral atmosphere with an altitude dependent secondary electron distribution and atmospheric distribution from a two-stream code, and (2) a H Ly- $\alpha$ line. The EUV ( $0.6 \AA$ per channel) and FUV (0.8 A per channel) data are smoothed by a 5-channel box-car smooth prior to merging. The model (2.4 Å FWHM with $0.6 \AA$ A channels) is smoothed by a 5-channel box-car smooth. (B) The Cassini FUV portion of the UV spectrum in units of $\mathrm{kR} / \AA$ A for the southern aurora of $29 \mathrm{Dec} 00$ fitted in linear regression analysis.

There is strong evidence of absorption by vibrationally excited $\mathrm{H}_{2}$ at $1050 \AA\left(v^{\prime \prime}=1\right)$ and $1100 \AA\left(v^{\prime \prime}=2\right)$ as there was for the HUT observations. The vibrationally excited $\mathrm{H}_{2}$ in the ground state is produced by excitation of the lowest vibrational levels by low-energy electron impact from secondary electrons and by excitation of all vibrational levels by emission from the Rydberg states (Waite et al., 1983). Wolven and Feldman (1998) have noted the strong effects of self-absorption near 1054 and $1100 \AA$ from the weak measured intensities of the $\mathrm{C}-\mathrm{X}(0,1)$ and $\mathrm{C}-\mathrm{X}(0,2)$ Werner band rotational lines, respectively. The optically thin model employed here predicts too much intensity in the neighborhood of 1054 and $1100 \AA$ for the 29Dec00 spectra. The synthetic spectral model does not include self-absorption by vibrationally excited molecules (only $v^{\prime \prime}=0$ is included). The long wavelength re-emission in the $v^{\prime \prime}>0$ fluorescence bands is a small effect on the relative intensities. Laboratory spectra of electron-excited $\mathrm{H}_{2}$ for wavelengths above $1100 \AA$, where the strong fluorescence from the Lyman and Werner band systems with $v^{\prime \prime}>0$ occurs (see band identification Table 1 in Ajello et al., 1984), does not show any change in the relative intensities with $\mathrm{H}_{2}$ gas pressure to column densities of $10^{15} \mathrm{~cm}^{-2}$ (see laboratory spectrum in Gustin et al., 2004). Optical depth effects in $v^{\prime \prime}=0$ become important at $10^{12} \mathrm{~cm}^{-2}$ (Jonin et al., 2000). The model also does not include a vector due to the weak $\mathrm{Ly}-\beta$ line near $1025 \AA$ A. The model fits of the 02Jan01 and 29Dec00 data sets are very similar in the FUV, since they have nearly identical FUV color ratios. However, there are discrepancies between the two data sets in the EUV. For the 29Dec00 auroral spectrum the strongest peak intensity in the EUV occurs at $1174 \AA(\mathrm{B}(9,4)$ and $\mathrm{B}(12,5)$ vibrational bands), while for the 02Jan01 auroral spectrum the strongest peak intensity is found at $1117 \AA \mathrm{C}(1,3)$ with no indication of selfabsorption from vibrationally excited $\mathrm{H}_{2}$ at $1025 \AA \mathrm{C}(1,1)$, $1054 \AA \mathrm{C}(0,1)$, and $1100 \AA \mathrm{C}(0,2)$. The absence of strong self-absorption in the rovibronic lines of the $\mathrm{C}\left(v^{\prime}, 1\right)$ and $\mathrm{C}\left(v^{\prime}, 2\right)$ progression may indicate either cooler temperatures (vibrational and gas) of $\mathrm{H}_{2}$ in the thermosphere (29Dec00 vs 02Jan01) or a stronger soft electron component for 02Jan01. This latter conclusion seems to be the case as the discussion below will demonstrate.

The 29Dec00 EUV and FUV low resolution spectra were carefully merged at the wavelength minimum of $1168 \AA$ based on the model. We make use of the two-stream spectral model compared to the EUV and FUV spectra. We show in Fig. 6 the over-plot of the two data sets. It is evident that the FUV portion of the spectrum shown as a dashed line fits the model down to a wavelength of nearly $1165 \AA$. On the other hand, the EUV portion of the spectrum fits the model up to a wavelength of $1170 \AA$. For convenience we join the two spectra at $1168 \AA$ at a minimum point in each spectrum. The large discrepancy between the EUV and FUV spectral data curves near $1145 \AA$ occurs from the rapid loss of sensitivity of the FUV channel near its short wavelength limit by the decline in transmission of the $\mathrm{MgF}_{2}$ window.

We show in Fig. 7 a comparison of three auroras (the two UVIS observations on 29Dec00 and 02Jan01 (except for 29Dec00 north) and the HUT 1995 observation). The spectral structure contrasting the EUV and FUV spectral regions is dramatically stronger than the normal FUV color ratio. We define a new color ratio, $\left(4 \pi I_{1550-1620 \AA} / 4 \pi I_{1030-1150 \AA}\right)$, contrasting the long wavelength FUV region (1550-1620 $)$ ) as in the normal color ratio with the EUV region defined 


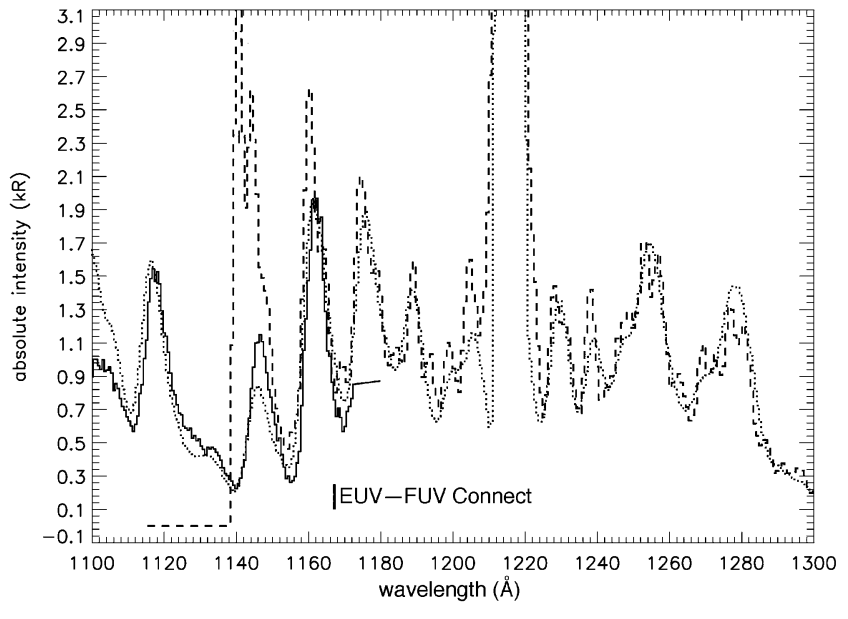

Fig. 6. The merging of the EUV (solid) and FUV (dash) auroral spectra of 29Dec00 near $1170 \AA$ A by comparison to the auroral model (dot).

from 1030 to $1150 \AA$. The FUV color ratio for the four observations varies from 2.2 to 2.7. A larger FUV color ratio indicates a higher primary electron characteristic energy. The EUV color ratio varies between 1.5 and 4.4; the ratio is a measure of the ratio of the hard to soft electron flux magnitudes. The 29Dec00 north observation that is not shown in Fig. 7, has an EUV color ratio of 2.0 and an FUV color ratio of 2.0. A low EUV color ratio is indicative of a large flux of soft electrons or a small flux of hard electrons or both. On a relative basis the $02 \mathrm{Jan} 01$ southern aurora data had a larger source of soft electrons or/and a smaller source of hard electrons then the $29 \mathrm{Dec} 00$ southern aurora data three days earlier. The EUV portion of the 02Jan01 spectrum is the most optically thin against $\mathrm{H}_{2}$ self-absorption of the three cases shown.

\section{HST observations}

We will focus on two sets of observations obtained by HST during the CC. There was a low-resolution G140L observation on December 28, 1999 (28Dec00) of the south polar region, and a medium-resolution G140M observation on January 13, 2000 (13Jan01) of the north aurora. The first observation coincided closely with the closest approach of Cassini on December 30, 2000. The 13Jan01 image was shown earlier in Fig. 1 and corresponded to the only opportunity to get a medium-resolution spectrum of the Jupiter aurora during the $\mathrm{CC}$.

\subsection{Medium-resolution STIS observations}

As obtained in 1999 (Gustin et al., 2002) and again during the CC, STIS obtained spatially resolved spectra of the po-
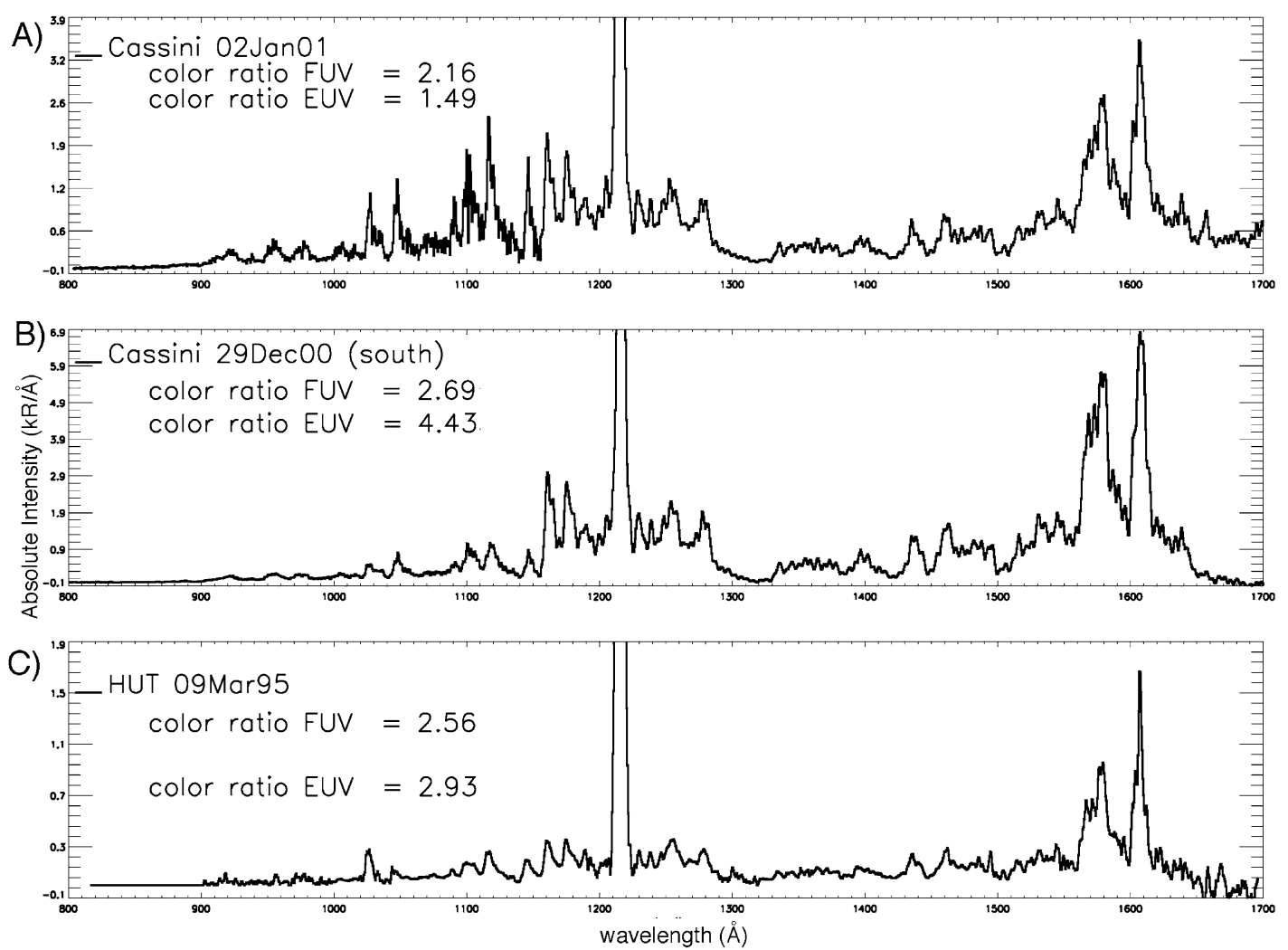

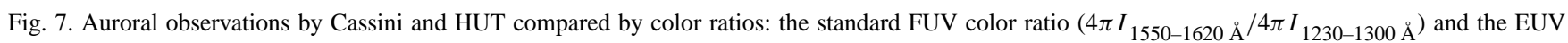
color ratio ( $4 \pi I_{1550-1620 \AA} / 4 \pi I_{1030-1150 \AA}$ ). The observations are: (A) 02 January 2001 (south), (B) 29 December 2000 (south), and (C) HUT 09 March 1995 (north). 
lar cap, aurora, limb and foot of Io flux tube. A narrow STIS aperture was chosen for both sets of observations. It measures $52 \times 0.5 \operatorname{arcsec}^{2}$ with the G140M grating corresponding to a spectral resolution of $\sim 0.9 \AA$. Two spectra were obtained with the wavelength band-pass of $50 \AA$ centered at 1321 and $1518 \AA$. The first wavelength band was set to span the wavelengths of the strongest $\left(\mathrm{B}^{1} \Sigma_{\mathrm{u}}^{+} \rightarrow \mathrm{X}^{1} \Sigma_{\mathrm{g}}^{+}\right.$) cascade bands arising by the rovibronic (rotational-vibrationalelectronic) transitions of EF, GK, $\mathrm{H} \overline{\mathrm{H}}, \ldots{ }^{1} \Sigma_{\mathrm{g}}^{+} \rightarrow \mathrm{B}{ }^{1} \Sigma_{\mathrm{u}}^{+}$. The resolution is sufficient to separate the effects of direct excitation and cascading contributions to individual rotational lines. The excitation function for each process has a different energy dependence and from the modeled ratio we can estimate the contributions of each process to the aurora thereby establishing the mean secondary electron energy present in the aurora.

The pumping of the low-lying vibrational levels of the B state by the many rovibronic cascade transitions produced by the Rydberg series of gerade states fashions an entirely different UV spectrum than the direct excitation spectrum (Dziczek et al., 2000). We show the two types of transitions that populate the B state in Fig. 8. The strongest cascade bands lie near $1335 \AA$ and arise from the $v^{\prime}=0$ progression. The difference in the internuclear separation for the $\mathrm{B}$ ${ }^{1} \Sigma_{\mathrm{u}}^{+}$state, the upper state of the Lyman bands and the first minimum of the EF ${ }^{1} \Sigma_{\mathrm{g}}^{+}$state, the upper state of the EF-B cascade bands accounts for the difference in the strongest $v^{\prime}$ progression. These vibrational levels are $v^{\prime}=7$ for direct excitation to the $\mathrm{B}$ state and $v^{\prime}=0$ for cascade to the $\mathrm{B}$ state. The strongest member of the progression is the $\mathrm{B}(0,4)$ band with rotational lines lying between 1330 and $1335 \AA$ A. The second region (near $1518 \AA$ ) for the STIS observation is populated mainly by direct excitation. It is also a region that has negligible methane absorption. We show the data and the model fits to the two spectral regions in Figs. 9A and 9B. Fig. 9A shows the short wavelength region with the regression model fit individual vectors. Fig. 9B shows the regression model fit in the long wavelength region. The best-fit rotational temperature occurs at $450 \mathrm{~K}$. Spectral models were generated for 300,450 , 600, and $900 \mathrm{~K}$ enabling us to minimize the regression model on the basis of temperature. The STIS spectrum in Fig. 9A shows the strongest rotational lines in the spectrum occur at $1334.0 \AA$ $\left(R_{0}, R_{1}\right), 1335.8 \AA\left(P_{1}, R_{2}\right)$, and $1342.1 \AA\left(P_{2}\right)$ the $\mathrm{B}(0,4)$ vibrational band. These rotational lines have roughly equal contributions from direct excitation and cascade. Fig. 9A plots the three independent spectral vectors that are used to complete the regression model of the data. These vectors are the EF cascade spectrum, the B direct spectrum, and the atomic $\mathrm{O}$ spectrum from the resonance features at 1302.2, 1304.9, and 1306.0 $\AA$. The atomic features arise because of the Earth's airglow detection at the altitude of the HST orbit. The inexact model fit below $1320 \AA$ may be also due to the presence of atomic and molecular nitrogen emission telluric features in the spectrum (Ajello et al., 1989;

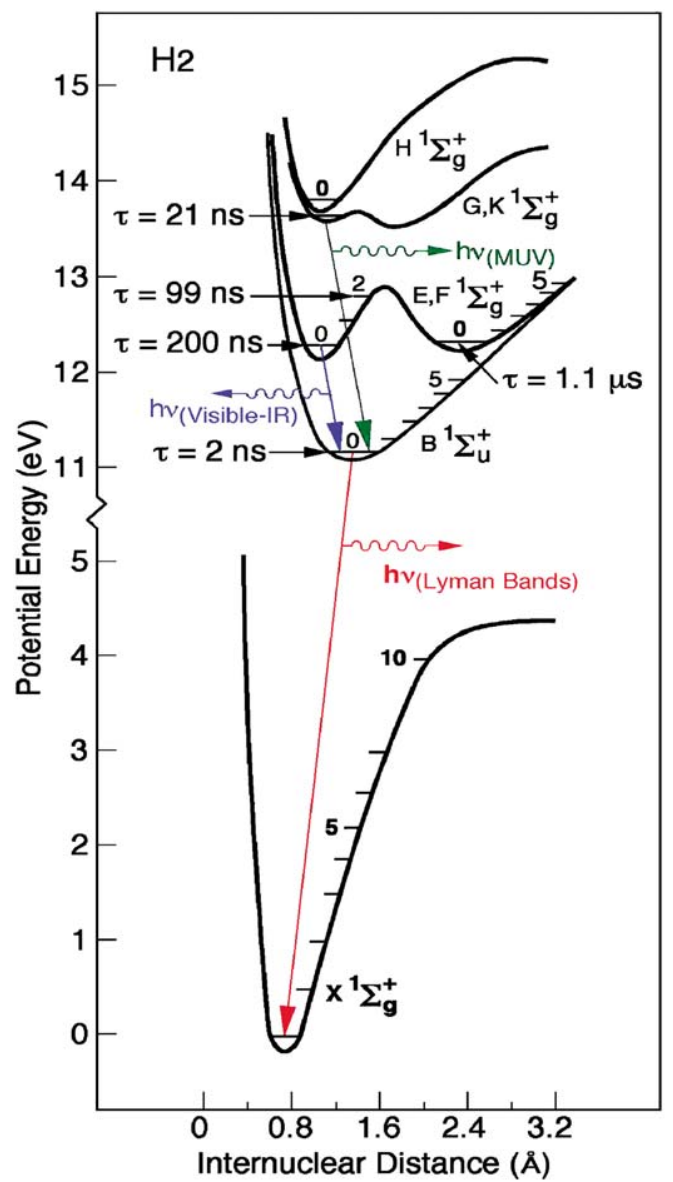

Fig. 8. A partial energy level diagram of $\mathrm{H}_{2}$ showing: (1) the two main Rydberg series (gerade and ungerade), the cascade transitions from the EF ${ }^{1} \Sigma_{\mathrm{g}}^{+}$gerade series to B ${ }^{1} \Sigma_{\mathrm{u}}^{+}$and (3) the life-times of the various vibrational transitions to indicate allowed and forbidden nature of the transitions (adopted from Dziczek et al., 2000). The cascade bands are observed in the visible and infrared.

Ajello and Shemansky, 1985, and references therein) from the Earth dayglow, unaccounted for in the model. For example there are atomic nitrogen lines at 1311, 1313, 1315, 1316, and $1320 \AA$. The model used to derive the spectrum consists of a slab emission layer of $\mathrm{H}_{2}$ with overlying methane absorption. The most important part of the STIS spectrum for estimating cascade effects lies between 1320 and $1345 \AA$.

The cascade model includes the full set of cascade spectra to the $\mathrm{B}^{1} \Sigma_{\mathrm{u}}^{+}, \mathrm{C}^{1} \Pi_{\mathrm{u}}, \mathrm{B}^{\prime}{ }^{1} \Sigma_{\mathrm{u}}^{+}$, and $\mathrm{D}^{1} \Pi_{\mathrm{u}}$ ungerade states from the $\mathrm{EF}^{1} \Sigma_{\mathrm{g}}^{+}$Rydberg series of gerade states. Most of the cascade ( $>95 \%$ ) undergoes transitions to the vibrational levels of the B-state (Dziczek et al., 2000). The direct excitation EUV and FUV synthetic line spectrum model of the $\mathrm{H}_{2}$ molecule has also been previously derived (Liu et al., 1995; Jonin et al., 2000). The regression model returns fitting coefficients with the contribution of each of these two independent spectra. The coefficients give the best fit contributions of these two spectra to model the data over the spectral range of 1295-1345 $\AA$. Based on the short wavelength range stud- 

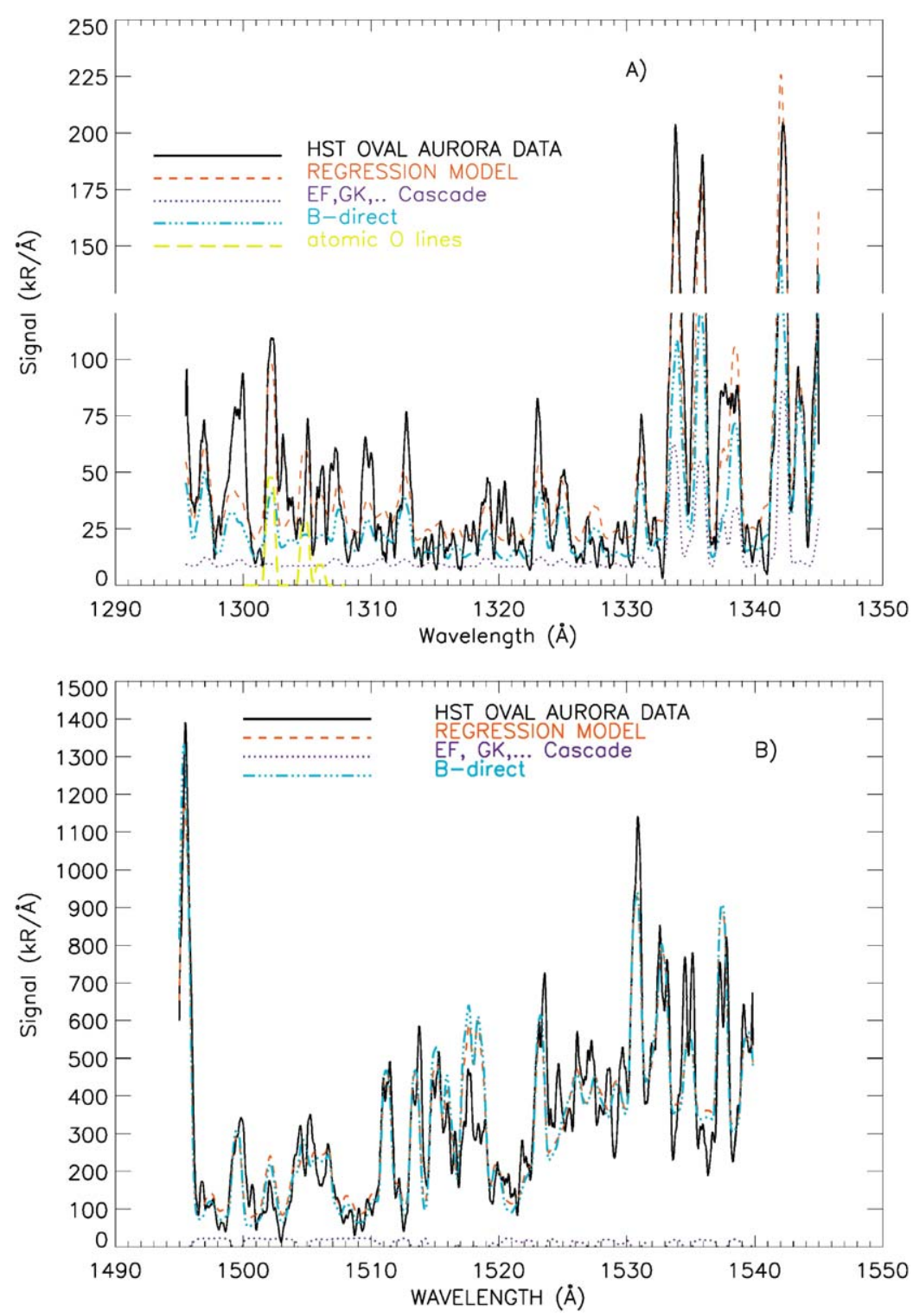

Fig. 9. (A) The HST STIS G140M relative photon intensity short wavelength spectrum (1295-1345 $\AA$ ) for the north aurora of 13Jan01 starting at 16:58:07 with a 480-s integration fitted in linear regression. The linear regression analysis with independent vectors of: (1) a direct excitation optically thin spectrum of the Rydberg bands of $\mathrm{H}_{2}$ by a monoenergetic electron distribution at $100 \mathrm{eV}$, (2) a cascade excitation optically thin spectrum of the Rydberg bands of $\mathrm{H}_{2}$ by a monoenergetic electron distribution at $100 \mathrm{eV}$ and (3) an atomic oxygen multiplet at $1304 \AA$. The regression model to the observational data is based upon a linear regression analysis with these three independent vectors and transmission through a slab of $\mathrm{CH}_{4}$. (B) The HST STIS G140M relative (photon) intensity for the medium wavelength spectrum (1495-1545 $\AA$ ) for the north aurora of 13Jan01 starting at 17:13:50 with a 480-s integration fitted in linear regression. The STIS data is smoothed with a 7-point box-car smooth; the data point step-size is $50 \mathrm{~m} \AA$.

ied, it is possible to estimate the total contributions from direct excitation and cascading. We find that $11 \%$ of the total Lyman band emission arises from cascading and $89 \%$ from direct excitation. To our knowledge this is the first estimate of the cascade contribution to the Lyman band Jupiter aurora. Thus the EF, GK, and $\mathrm{H} \bar{H}$ total cascade cross-section is the third strongest excitation process of emission by the $\mathrm{H}_{2}$ Rydberg series of ungerade states. The two most important emission cross-sections are the Lyman (B-X) and Werner $(\mathrm{C}-\mathrm{X})$ band cross-sections from direct excitation (Jonin et al., 2000). Most of the cascade emission occurs in the FUV. The fourth strongest band system emission cross-section is the $\mathrm{B}^{\prime}-\mathrm{X}$ band system with an emission cross-section of $8 \%$ of the $\mathrm{B}-\mathrm{X}$ direct excitation cross-section. This band system lies in the EUV (850-1150 ̊).

The energy dependencies of the cascade cross-section and direct excitation for the Lyman bands are different. Direct excitation is dipole-allowed with the high-energy Born behavior and a peak cross-section energy of $50 \mathrm{eV}$ (Liu et al., 1998). The cascade cross-section is optically forbidden and has been described (Dziczek et al., 2000; Liu et al., 2003). In Dziczek et al. (2000) we find that the cascade cross-section falls by a factor of 3.4 between 20 and $100 \mathrm{eV}$ with a peak cross-section between 15 and $20 \mathrm{eV}$. 


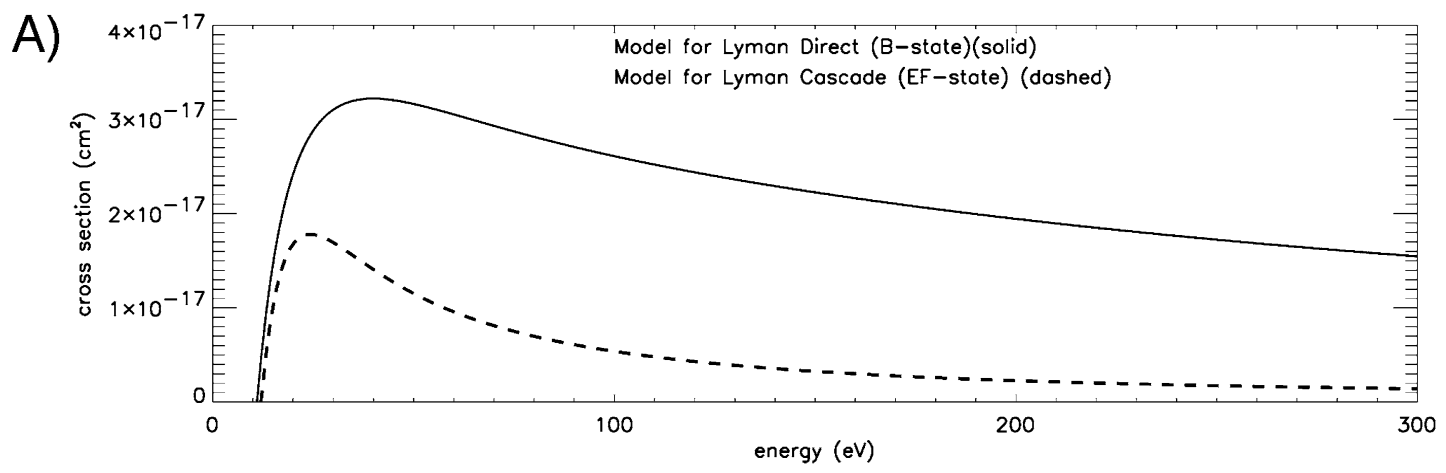

B)

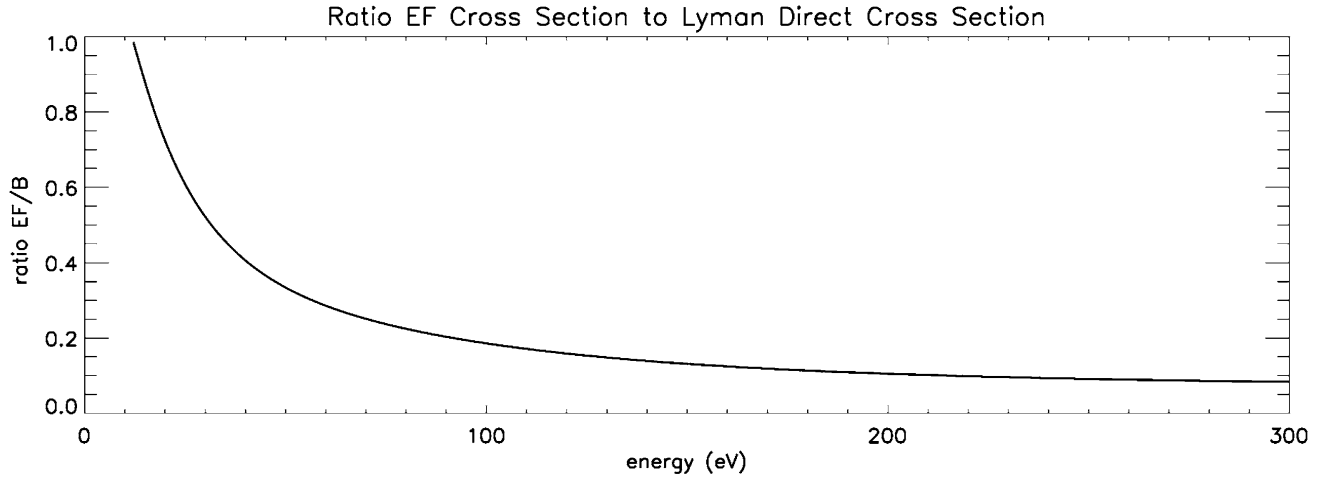

Fig. 10. (A) A plot of the Lyman band direct and excitation cross-sections from (Liu et al., 1998; Dziczek et al., 2000, and Liu et al., 2003). (B) A plot of the ratio $(\mathrm{EF} / \mathrm{B})$ of the cross-sections from $(\mathrm{A})$.

We plot in Fig. 10 the cross-sections (top) and the ratio of the cross-sections $(\mathrm{EF} / \mathrm{B})$ for these two processes (bottom). The ratio of the integrated intensities in Fig. 10A from each process is equal to the ratio of the cross-sections at the mean secondary electron energy. The measured ratio in Fig. 10A is $12 \%$ corresponding to a mean secondary electron energy of $175 \mathrm{eV}$. We have re-analyzed the 1999 STIS G140M aurora, polar cap, and limb data (Gustin et al., 2002) in the same way and find that the EF contribution for these observations varies between 10 and $20 \%$ (EF/B ratio $0.11-0.25$ ) corresponding to a mean secondary electron energy of 50$200 \mathrm{eV}$.

The long wavelength region of the STIS spectrum shown in Fig. 9B gives an excellent indication of the $\mathrm{C}_{2} \mathrm{H}_{2}$ column density from its strong absorption band near $1520 \AA$. The model does not account for all of the absorption away from $1520 \AA$. We estimate the column density of $\mathrm{C}_{2} \mathrm{H}_{2}$ to be $2 \times 10^{15} \mathrm{~cm}^{-2}$. This column density compares favorably with column densities of $\mathrm{C}_{2} \mathrm{H}_{2}$ estimated in Ajello et al. (1998). The other distinct regions of the aurora, the polar cap and limb, observed on 13Jan01 will be discussed in a forthcoming paper (Gustin, private communication).

\subsection{The December 28, 2000 HST STIS observation}

The image/spectrum pair lying closest in time to the Cassini closest approach occurred on 28Dec00 at 07:15 UT. We show the linear regression fit to the data in Fig. 11. The model in Fig. 11 is based on the Grodent two-stream electron distribution (Grodent et al., 2001) and the $\mathrm{H}_{2}$ modeling code as described in Ajello et al. (2001). The modeling result shows that the energy input to the aurora is approximately $10 \mathrm{erg} / \mathrm{cm}^{2} / \mathrm{s}$ with a characteristic energy in the primary electron distribution of $22 \mathrm{keV}$ ( $44 \mathrm{keV}$ mean energy). The measured integrated intensity (1150-1700 $\mathrm{A}$ ) is $49 \mathrm{kR}$ for the measured FUV spectrum.

\section{Discussion}

The Cassini UVIS obtained a large set of instrument performance and science observations during the Jupiter flyby in preparation for the Saturn encounter in 2004. Of fundamental interest is the fact that the auroral observations at Jupiter indicate the EUV wavelength range (800-1100 A) are very sensitive to variations in the soft electron flux $(\varepsilon \sim 100-3000 \mathrm{eV})$. The variation in the soft electron flux of primary electrons incident on the jovian atmosphere can be described by an EUV color ratio. The EUV color ratio is found to vary by nearly a factor of three for observations that do not contain contributions from the dayglow. This variation may indicate a sharp change in the ratio of the fluxes of the hard to soft electrons. Grodent et al. (2001) show that there are three electron energy deposition peak altitudes in the atmosphere corresponding to the three magnitudes of characteristic energies in the model: weak $(100 \mathrm{eV})$, soft ( $3 \mathrm{keV}$ ), and hard (22 keV for a discrete aurora). The altitudes of the peak emission layers are 250,600 , and $1500 \mathrm{~km}$ approximately (see Fig. 6 of Grodent et al., 2001). The altitude evolution of the auroral energy distribution from $1 \mathrm{eV}$ 


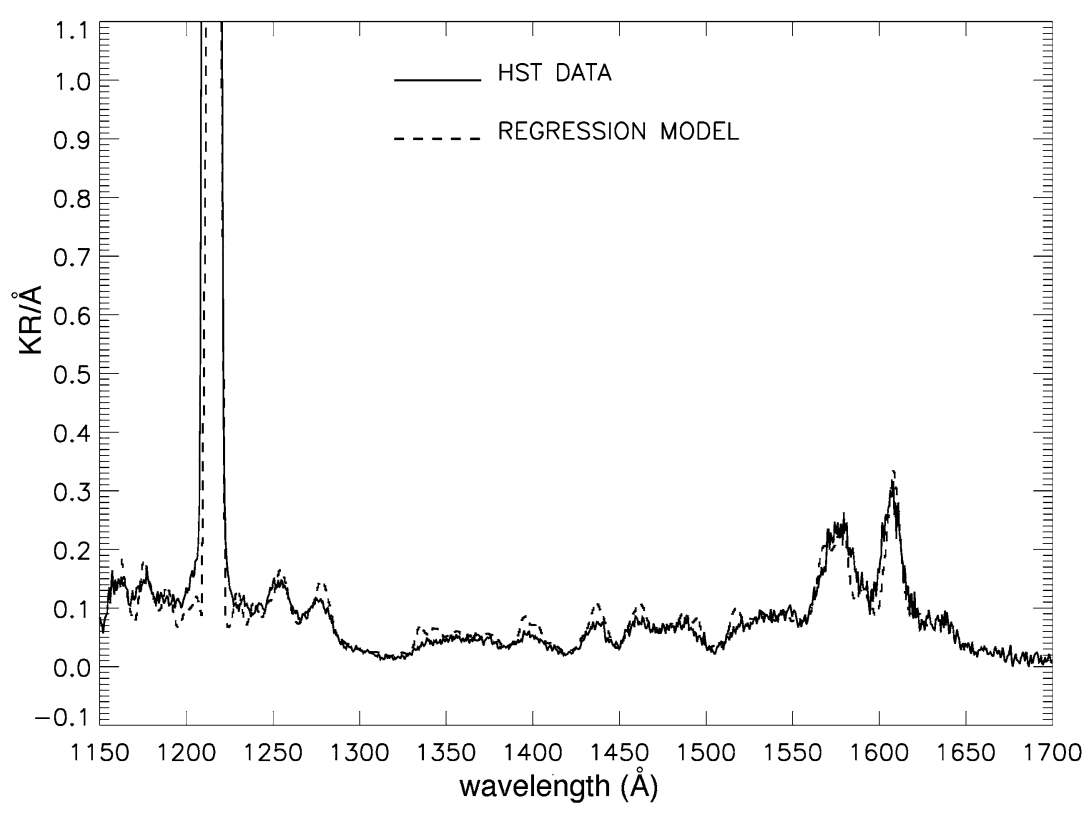

Fig. 11. The HST G140L FUV spectrum in units of kR/ $\AA$ for the south aurora of 28 December 2000 (start time = 07:15:22, 630 s integration) fitted in linear regression analysis with: (1) a discrete aurora electron flux (Grodent et al., 2001) incident upon an auroral atmosphere with an altitude-dependent secondary electron distribution and atmospheric distribution from a two-stream electron transport code, and (2) a H Ly- $\alpha$ line.

to $1 \mathrm{MeV}$ is shown in Fig. 2 of Grodent et al. (2001). The weak and soft electron peaks are found above the homopause where no $\mathrm{CH}_{4}$ absorption occurs (Ajello et al., 1998). Occasionally, the Galileo UVS observed aurora with no $\mathrm{CH}_{4}$ absorption, indicating no hard electron energy component. The EUV emission observed by a spacecraft is produced, predominantly, from a region just above the homopause (400$1000 \mathrm{~km}$ ) as shown in Fig. 7b of Ajello et al. (2001). The EUV color ratio is more sensitive to the soft component, $3000 \mathrm{eV}$ than the weak component, $100 \mathrm{eV}$, whose emission peak lies near the exobase. The electron energy distribution model fits to the UVIS EUV spectral data may not be unique. Other electron distribution functions of the weak and soft electron components may provide excellent fits to the data (cf. models of the HUT observation by Ajello et al., 2001 and Gustin et al., 2004).

We have modeled UVIS spectra taken at closest approach, 29Dec00 and 02Jan01. The spectra were modeled with a two-stream electron transport code found in Grodent et al. (2001). Grodent et al. (2001) have chosen an auroral emission model, which is generated by the influx of a primary electron flux consisting of three components with characteristic energies $E_{0}: 100 \mathrm{eV}, 3 \mathrm{keV}$, and $15 \mathrm{keV}$ (diffuse) and $22 \mathrm{keV}$ (discrete). We have modeled the data shown in the model-data comparisons considered with the discrete case. Both models are capable of fitting most of the observations with no additional soft electron flux requirements, since they are already included in the initial Grodent et al. electron distribution function model. The high-energy component is a kappa distribution for the diffuse aurora case and the two low-energy components are combined as a double Maxwellian. We have also shown that a $25 \mathrm{keV}$ Maxwellian with a kappa distribution of kappa index 2.1 and characteris- tic energy $15 \mathrm{keV}$ will be stopped very near $250 \mathrm{~km}$ (Ajello et al., 2001; Vasavada et al., 1999). The results indicate that the electron distribution chosen by Grodent et al. (2001) is an excellent model capable of reproducing the auroral emissions measured by Cassini UVIS on 02Jan01 in the south, the 29Dec00 in the north, and the 28Dec00 STIS observation in the south. However, the Grodent et al. primary electron distribution is not able to model the 29Dec00 southern aurora data shown in Fig. 7B. The FUV color ratio of 2.69 requires a higher characteristic electron energy $(30-50 \mathrm{keV})$ similar to the HUT observation of Fig. 7C.

An important question arises as to whether the aurora spectral characteristics change on a timescale of tens of minutes as the planet rotates beneath the Cassini UVIS boresight. We ask this question since the 02Jan01 observation had a duration of $4.5 \mathrm{~h}$ (nearly half a rotation); and the observation has been analyzed on the basis of the average spectrum shown in Fig. 4. We have broken the observation into six separate observations of $31.3 \mathrm{~min}$ (20 spectral records of $94 \mathrm{~s}$ per record). Each observation group, is referred to by an observation number from 0 to 5 . Each observation is separated by $47 \mathrm{~min}$. The planet rotated a total of $166^{\circ}$ during the entire observation with about $18^{\circ}$ per individual spectrum. The relative spectral characteristics of the EUV channel and FUV channel varied strongly over this period. We show a plot of the EUV and FUV relative intensities in Fig. 12A and a plot of the EUV and FUV color ratios in Fig. 12B as a function of observation number. The variation of integrated intensities in the FUV was a factor of eight and in the EUV a factor of three. The peak-integrated signal for each channel occurred at a different observation group number. The peak in EUV signal occurred during observation period 4 from time $141 \mathrm{~min}$ to $172 \mathrm{~min}$, whereas the peak 

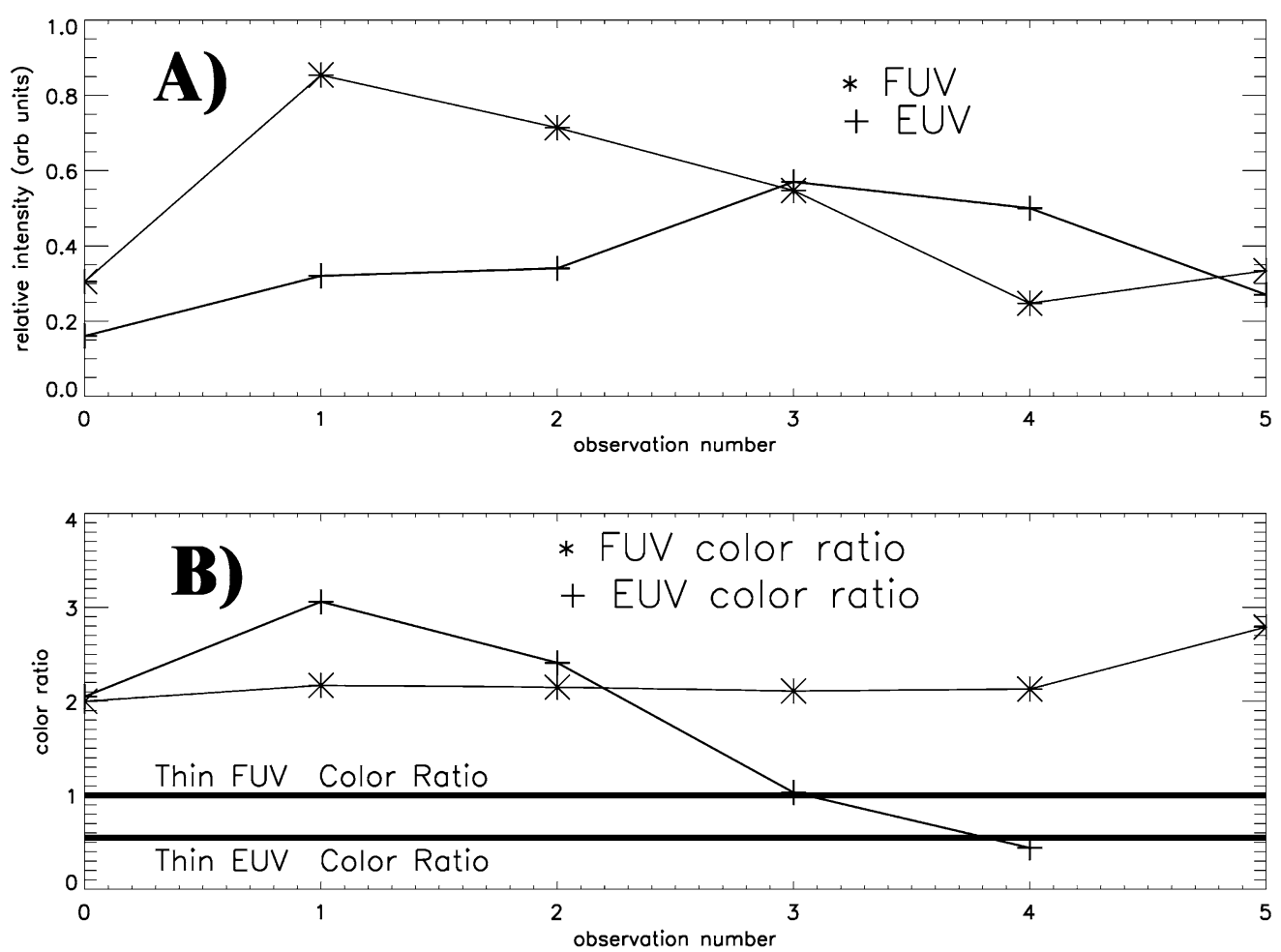

Fig. 12. (A) The relative peak intensities (exclusive of $H$ Ly- $\alpha$ ) of the EUV and FUV channels for the six observation time intervals of 02 Jan01. The 02Jan01 observation of $4.62 \mathrm{~h}$ is subdivided into six equal time interval of $47 \mathrm{~min}$, each with an integration time of $31.3 \mathrm{~min}$. The start times relative to the beginning of the observation in minutes from 06:03 UT are 0, 47, 94, 141, 188, and $225 \mathrm{~min}$ from the start of the observation. The abscissa is labeled observation number according to the start time with observation 0 referring to the start time of $0 \mathrm{~min}$. The integrated intensities for the EUV were measured from 1030 to $1150 \AA$ A. The integrated intensities for the FUV were measured from 1500 to $1700 \AA$. (B) The EUV and FUV color ratios for observations $0-4$. The S/N for observation 5 was insufficient to get a statistically significant EUV color ratio. The plot also shows the optically thin EUV and FUV color ratios from a laboratory experiment. The experiment was conducted at $100 \mathrm{eV}$ electron impact energy with the same spectral resolution of $2.4 \AA$ as the Cassini UVIS (see Jonin et al., 2000). The color ratio for integrated intensities for EUV was defined $\left(4 \pi I_{1550-1620 \AA} / 4 \pi I_{1030-1150 \AA}\right)$. The color ratio for integrated intensities for FUV was defined $\left(4 \pi I_{1550-1620 \AA} / 4 \pi I_{1230-1300 \AA)}\right.$.

FUV signal occurred during observation period 2 from 47 to $78 \mathrm{~min}$. The FUV color ratio was nearly constant varying from 2.00 to 2.16 for the first 188 min. The last observation period with a minimal of counts had a color ratio that was slightly more. The variation in the EUV color ratio is very striking as it varied a factor of 6 for the observations 0-4. The last point, observation 5, for the EUV color ratio plot is uncertain and is not shown. For comparison to a laboratory benchmark color ratio we conducted a low pressure optically thin laboratory experiment (see references in Liu et al., 2002) with the Cassini spectral resolution of $2.4 \AA$ FWHM and at $100 \mathrm{eV}$ electron impact energy, a value near the mean secondary electron energy found earlier. We find that the optically thin color ratios are 0.55 for the EUV and 1.0 for the FUV. The FUV color ratio measured during the CC always shows the effects of methane absorption and never approaches the laboratory value. On the other hand the Galileo ultraviolet spectrometer observations measured color ratios with no apparent methane absorption (Ajello et al., 1998). However, the EUV color ratio can swing from nearly optically thin to optically thick (weak intensity of soft electrons as in observation 1, Fig. 12B) as the aurora rotates beneath the UVIS boresight.
The meaning of the extreme variations for the EUV color ratio for this period are found by comparing the full UV spectrum for observations 1 and 3 in Figs. 13A and 13B, respectively. The observation 3 model requires a contribution from an additional source of soft electrons, by an energy flux amount that exceeds the relative soft electron flux in the Grodent et al. (2001) electron distribution model. The ratios of high-energy $(\sim 22 \mathrm{keV})$ to low-energy electron energy flux $(\sim 3000 \mathrm{eV})$ and weak electron flux $(\sim 100 \mathrm{eV})$ are fixed at 2 and 40, respectively, in the Grodent et al. (2001) model. One way this low-energy contribution can be made available would be to include an optically thin laboratory spectrum, 20-3000 eV in the synthetic model code. We arbitrarily include a $20 \mathrm{eV}$ laboratory spectrum as an added vector in the regression analysis in Fig. 13.

Observation 1 indicates a deep aurora with self-absorption within the $v^{\prime \prime}=1$ and $2, v^{\prime}$ progressions. Note the inability of our model, which does not include self-absorption for $v^{\prime}>0$ to fit the observation 1 data for wavelengths at 1054 and $1100 \AA$. The peak signal in observation 1 is $1174 \AA$, indicating a deep aurora without significant contribution from low-energy electrons. The peak signal in observation 3 is $1117 \AA$ showing a strong con- 

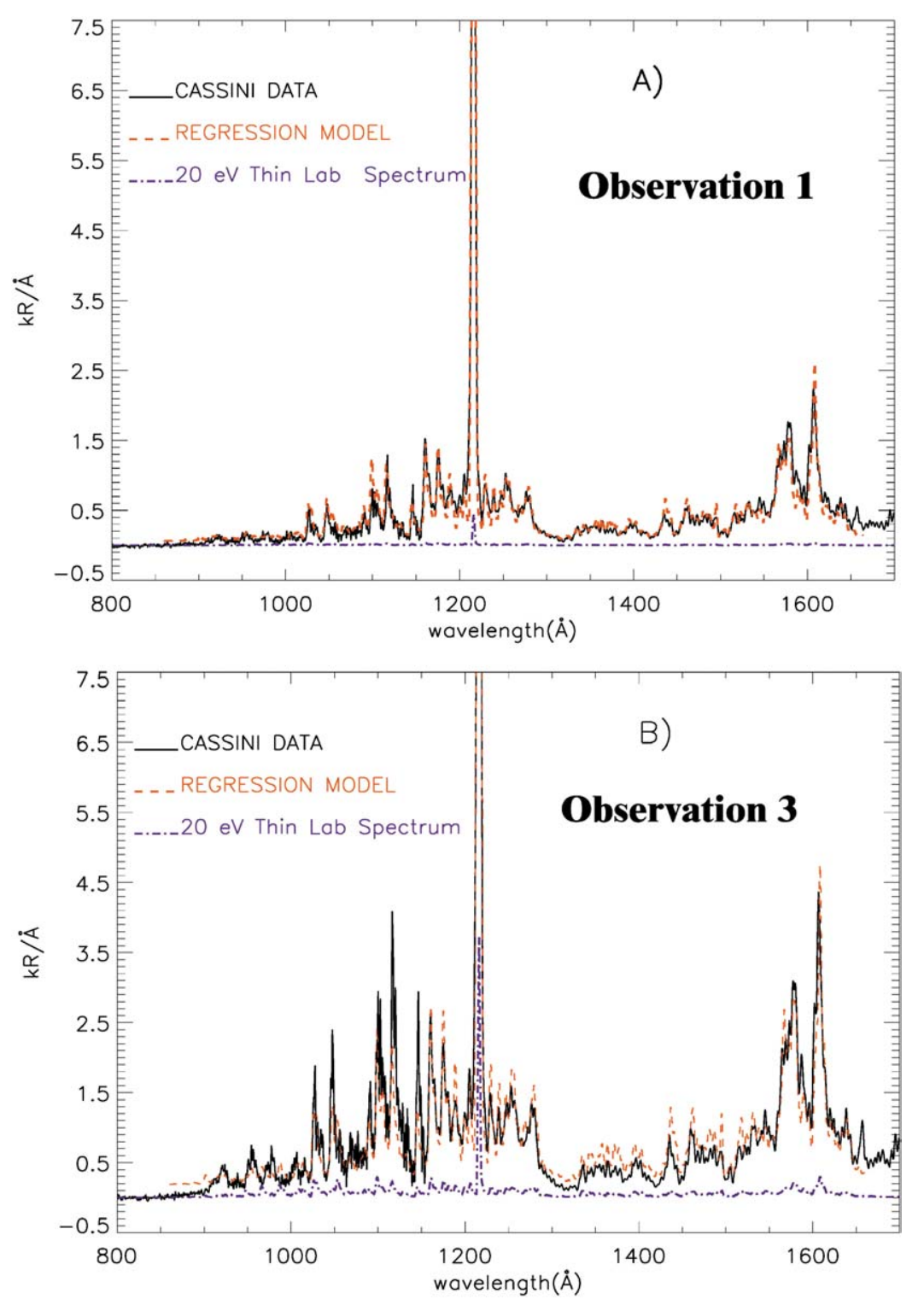

Fig. 13. (A) Observation 1 from 02Jan01 showing the combined EUV + FUV spectrum that started at 47 min after the observation start time in Table 1. The plot is a sum of 20 records of $94 \mathrm{~s}$ each. The numbering of records is given as the $x$-axis in Figs. 12A and 12B. (B) Observation 3 from $02 \mathrm{Jan} 01$ showing the combined EUV + FUV spectrum that started at $141 \mathrm{~min}$ after the observation start time in Table 1. The plot is a sum of 20 records of $94 \mathrm{~s}$ each. We include a model regression of observations 1 and 3 which are fitted in a linear regression analysis with: (1) a discrete aurora electron flux (Grodent et al., 2001) incident upon an auroral atmosphere with an altitude-dependent secondary electron distribution and atmospheric distribution from a two-stream code, (2) a nearly optically thin $\mathrm{H}_{2}$ Rydberg band laboratory spectrum excited by a $20 \mathrm{eV}$ monoenergetic electrons, and (3) a $\mathrm{H}$ Ly- $\alpha$ line. We show the regression model fit to the observations with the $20-\mathrm{eV}$ component as discussed in the text.

tribution from a low-energy electron aurora in the thermosphere.

It appears that the main variation in the EUV color ratio as defined in this paper is a very sensitive indicator of auroral variations of the electron energy flux. The change occurs from strong variations in the flux level of both the low- and high-energy primary fluxes. Although both EUV and FUV integrated intensities vary, the FUV intensity has the greater variations as shown in Fig. 12A.

A more extended observation period of FUV observations occurred during the Galileo Mission. We show in Fig. 14 the FUV color ratios for the prime Galileo Mission 1996-1998.
There were 38 observations in the north and 28 observations in the south with sufficient $\mathrm{S} / \mathrm{N}$ for the color ratio study. The record numbers are given. We can show from the Galileo predawn observations of the main oval during the two years of observations that the color ratio of the north oval shown in Fig. 14 is highly stable (Ajello et al., 1998). The color ratio of the FUV spectrum measured by the Galileo UVS was nearly constant over the entire mission at a value of $1.5 \pm 0.5$ for the predawn auroral oval. We have parameterized this color ratio with the methane column density. A column density of $0-3 \times 10^{16} \mathrm{~cm}^{-2}$ for methane will reproduce all the FUV color ratios (values between 1 and 2) 

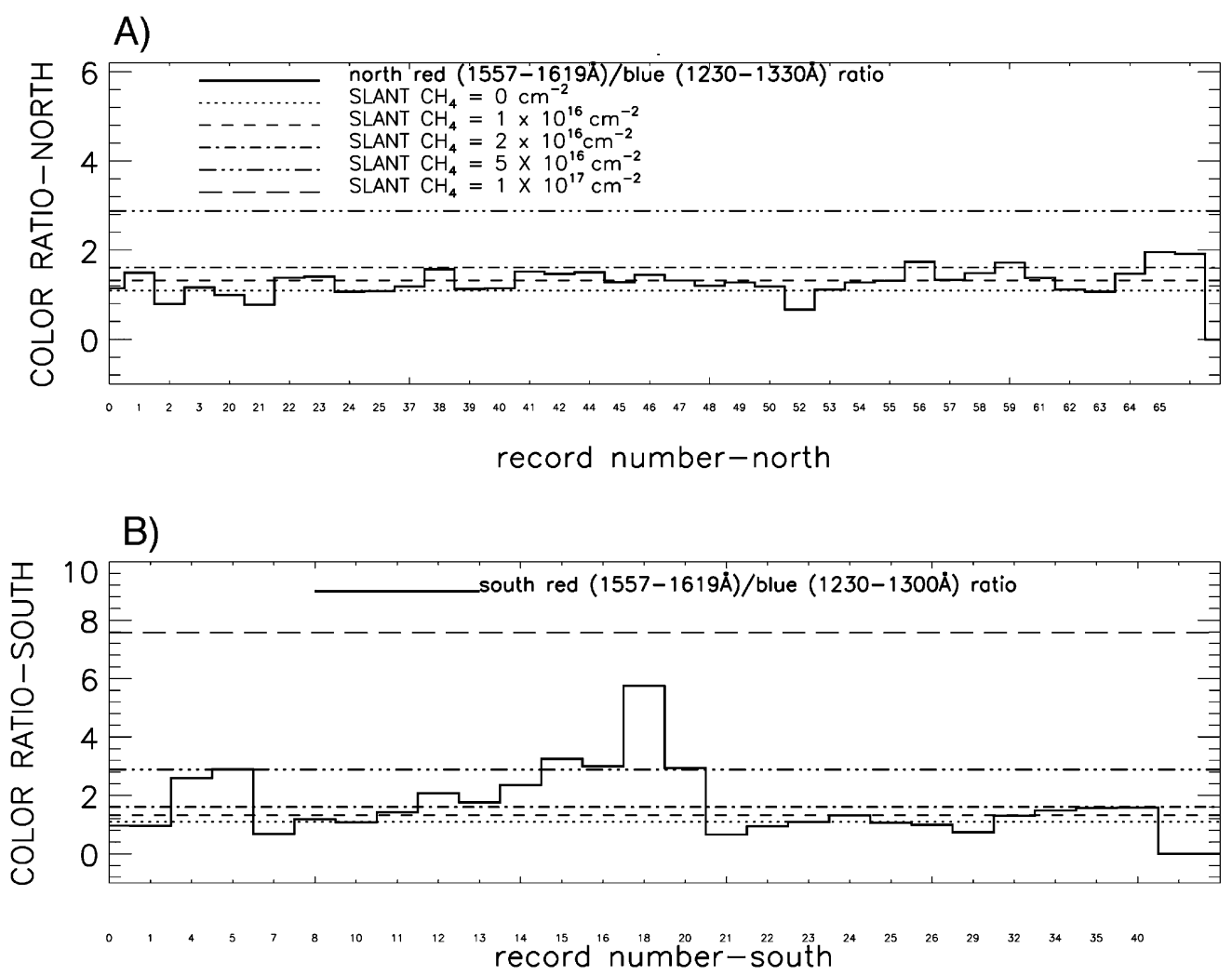

Fig. 14. A comparison of the color ratios for the north and south Galileo UVS FUV observations over the two-year prime mission (1996-1998). The record numbers are a measure of the time from orbits Ganymede 1 (DOY172-1996) through Europa 11 (DOY 309-1997). There were 3-5 observations every orbit.

in the north. The dispersion of the color ratio measurement is much larger in the south (1-5) corresponding to column densities of $0-10 \times 10^{16} \mathrm{~cm}^{2}$; though most color ratio calculations for the south tend to approach the lower values, similar to the north color ratios. We can compare IUE, STIS and Galileo color ratios and intensities. The Galileo color ratio results for the north are different from the IUE color ratios for the north (Livengood et al., 1990). Livengood et al. show in their Fig. 1 a large color ratio variation of 1-6 for the north auroral polar region. The IUE observations occur near local noon, whereas the Galileo observations were at local dawn. Local dawn is where HST spectra have occasionally shown large color ratio variations. The Galileo data was predawn and the measured spectra were acquired over many hours (Ajello et al., 1998). Recently, Gérard et al. (2002) confirmed the IUE results with their analysis of the STIS observations obtained over the period 1997-2001. Gérard et al. found a main oval variation of 1.4-10.4, also at local noon. The mean value of the FUV color ratio for IUE is 2.5 averaged over many years of observations (Harris et al., 1996). The three observations of Cassini shown in Fig. 7 have values very close to the IUE average. They occurred in the late afternoon local time. There is more than the difference of mean color ratios that distinguishes Galileo and IUE. IUE shows a dependence of color ratio on system III CML, whereas Galileo UVS has measured a color ratio that is independent of system III. The Galileo results seem to indicate that the average FUV color ratio in the north is constant in both space (system III longitude) and time for the predawn to morning terminator region. The average northern aurora observation by Galileo can be modeled by assuming a $25 \mathrm{keV}$ Maxwellian with kappa distribution with a characteristic energy of $15 \mathrm{keV}$ for the high-energy component of the primary electron flux (Ajello et al., 2001). Both the IUE and Galileo (not shown) spacecraft agree that there is a dependence of intensity on system III longitude. The Galileo data shows a peak intensity in the north near $180^{\circ}$ longitude and a peak intensity in the south between $0^{\circ}$ and $100^{\circ}$ longitude. Based on the indicated color variation with system III we can surmise that there may be a fundamental difference in the aurora primary electron flux between predawn and afternoon. Certainly the morphology of the aurora is different for these two periods. The predawn and morning terminator region is sharply defined by a bright arc with occasional dawn storms. The afternoon region is found to be more diffuse and less intense and a wider spread in latitude than the morning side region (Gérard et al., 2003; Clarke, 1998; Vasavada et al., 1999). The primary flux may vary with local time with a more temporally stable and lower characteristic energy in the predawn region.

Dols et al. (2000) studied eight G140L spectra from HST Goddard High Resolution Spectrograph (GHRS), near local noon, and found that the spectra from the oval had color ratios in the 1.9-2.7 region. Dols et al. also found that the high 
spatial resolution observations of the polar cap aurora show a much larger variation of 1.9-5.5. Gérard et al. (2002) have complemented the polar study with a larger data set and find a large range of variation with values up to 6.7. Galileo and Cassini average over tens of degrees in longitude, since the observation time may be $30 \mathrm{~min}$ or more for a color ratio determination ratio, compared to HST observation times of only a few minutes. The stability of the FUV aurora at local dawn should make this temporal difference unimportant for Galileo. Cassini UVIS has shown that the afternoon sector FUV color ratios were stable for the $4.5 \mathrm{~h} 02 \mathrm{Jan} 01$ observation.

A recent study of the CC with HST STIS using both $\mathrm{SrF}_{2}$ and clear filter FUV imaging of the polar cap aurora finds that the polar cap contributes $30 \%$ of the auroral signal (Grodent et al., 2003a). While the auroral oval remains steady the polar cap shows a transient behavior of short bursts lasting $\sim 100 \mathrm{~s}$. These short-term changes should not affect the long time averages presented here.

The G140M STIS aurora spectra reveal rovibronic structure that is dependent on gas temperature. Analysis of the fine structure of the STIS G140M FUV spectral observations can be greatly simplified if the Lyman system cascade and direct synthetic spectra are considered as two separate processes. The principal advantage is that the synthetic spectra from all Rydberg states (gerade and ungerade) become independent of energy (except near the threshold region $\sim 10-20 \mathrm{eV}$ ). The resulting spectrum from an admixture of $\mathrm{EF}$ (cascade) and B (direct) is still energy dependent because of the cross-section shape function differences. With this specialized analysis of the Lyman bands and using the recently measured cascade and direct excitation cross-sections we were able to show that the mean secondary electron energy producing the UV aurora is in the range $50-200 \mathrm{eV}$.

\section{Acknowledgments}

This work is one part of the Cassini UVIS investigation, supported by the NASA/JPL Cassini Mission. The Jet Propulsion Laboratory, California Institute of Technology Senior Research Scientist Sabbatical Program grant to J. Ajello supported this research. J. Ajello thanks the Laboratory of Atmospheric and Space Physics at the University of Colorado for providing the facilities and travel support to conduct this research. The STIS analysis is based on observations with the NASA/ESA Hubble Space Telescope, obtained at the Space Telescope Science Institute, which is operated by the AURA, Inc. for NASA. W. Pryor acknowledges support from the NASA Planetary Atmospheres Program and NASA/JPL. We thank X. Liu for providing the high-resolution EF cascade spectra. J.C. Gérard and D. Grodent are supported by the Belgium Foundation of Scientific Research (FNRS) and funded by a PRODEX HST contract of the European Space Agency.

\section{References}

Ajello, J.M., Shemansky, D.E., 1985. A re-examination of important $\mathrm{N}_{2}$ cross-sections by electron impact with application to the dayglow: The Lyman-Birge-Hopfield band system and NI (119.99 nm). J. Geophys. Res. 90, 9845-9861.

Ajello, J.M., Shemansky, D., Kwok, T.L., Yung, Y.L., 1984. Studies of extreme ultraviolet emission from Rydberg series of $\mathrm{H}_{2}$. Phys. Rev. A 29, 636-653.

Ajello, J.M., James, G.K., Franklin, B.O., Shemansky, D.E., 1989. Mediumresolution studies of EUV emission from $\mathrm{N}_{2}$ by electron impact: Vibrational perturbations and cross-sections of the $c_{4}{ }^{1} \Sigma_{\mathrm{g}}^{+}$and $b^{1} \Pi_{\mathrm{u}}$ states. Phys. Rev. A 40, 3524-3560.

Ajello, J.M., Shemansky, D., Pryor, W., Tobiska, K., Hord, C., Stephens, S., Stewart, I., Clarke, J., Simmons, K., McClintock, W., Barth, C., Gebben, J., Miller, D., Sandel, B., 1998. Galileo orbiter ultraviolet observations of Jupiter aurora. J. Geophys. Res. 103, 20125-20148.

Ajello, J.M., Shemansky, D.E., Pryor, W.R., Stewart, A.I., Simmons, K.E., Majeed, T., Waite, J.H., Gladstone, G.R., Grodent, D., 2001. Spectroscopic evidence for high altitude aurora at Jupiter from Galileo extreme ultraviolet spectrometer and Hopkins Ultraviolet Telescope observations. Icarus 152, 151-171.

Broadfoot, A.L., Sandel, B.R., Shemansky, D.E., McConnell, J.C., Smith, G.R., Holberg, J.B., Atreya, S.K., Donahue, T.M., Strobel, D.F., Bertaux, J.L., 1981. Overview of the Voyager ultraviolet spectrometry results through Jupiter encounter. J. Geophys. Res. 86, 8259-8284.

Bunce, E.J., Cowley, S.W.H., 2001. Divergence of the equatorial current in the dawn sector of Jupiter's magnetosphere: Analysis of Pioneer and Voyager magnetic field data. Planet. Space Sci. 49, 1089-1113.

Clarke, J.T., 10 colleagues, 1998. Hubble Space Telescope imaging of Jupiter's UV aurora during the Galileo orbiter mission. J. Geophys. Res. 103, 20217-20236.

Cowley, S.W.H., Bunce, E.J., 2001. Origin of the main oval in Jupiter's coupled magnetosphere-ionospheric system. Planet. Space Sci. 49, 10671088.

Dols, V., Gérard, J.C., Clarke, J.T., Gustin, J., Grodent, D., 2000. Diagnostics of the jovian aurora deduced from ultraviolet spectroscopy: Model and HST/GHRS observations. Icarus 147, 251-266.

Dziczek, D., Ajello, J., James, G., Hansen, D., 2000. A study of the cascade contribution to the $\mathrm{H}_{2}$ Lyman band system from electron impact. Phys. Rev. A 61, 4702-4706.

Emerich, C., Jaffel, L.B., Clarke, J.T., Prangé, R., Gladstone, G.R., Sommeria, J., Ballester, G., 1996. Evidence for supersonic turbulence in the upper atmosphere of Jupiter. Science 273, 1085-1087.

Esposito, L.W., 18 colleagues, 2004. The Cassini Ultraviolet Imaging Spectrograph investigation. Space Sci. Rev. 115, 299-361.

Gérard, J.-C., Gustin, J., Grodent, D., Clarke, J.T., Grard, A., 2002. The excitation of the FUV Io tail on Jupiter: Characterization of the electron precipitation. J. Geophys. Res. 107 (A11), 1394, doi:10.1029/2002JA009410.

Gérard, J.-C., Gustin, J., Grodent, D., 2003. Spectral observations of transient features in the FUV jovian polar aurora. J. Geophys. Res. 108 (A8), 1319, doi:10.1029/2003JA009901.

Grodent, D., Waite, J.H., Gérard, J.C., 2001. A self-consistent model of the jovian auroral thermal structure. J. Geophys. Res. 106, 12933-12952.

Grodent, D., Clarke, J.T., Waite, J.H., Cowley, S.W., Gérard, J.-C., Kim, J., 2003a. Jupiter's polar auroral emissions. J. Geophys. Res. 108 (A10), 1366, doi:10.1029/2003JA010017.

Grodent, D., Clarke, J.T., Kim, J., Waite, J.H., Cowley, S.W., 2003 b. Jupiter's main auroral oval observed with HST-STIS. J. Geophys. Res. 108 (A11), 1389, doi:10.1029/2003JA009921.

Gustin, J., Grodent, D., Gérard, J.C., Clarke, J.T., 2002. Spatially resolved far ultraviolet spectroscopy of the jovian aurora. Icarus 157, 91-103.

Gustin, J., 10 colleagues, 2004. Jovian auroral spectroscopy with FUSE: Analysis of self-absorption and implications on electron precipitation. Icarus 171, 336-355. 
Harris, W.M., Clarke, J.T., McGrath, M.A., Ballester, G.E., 1996. Analysis of jovian auroral H Ly- $\alpha$ emission (1981-1991). Icarus 124, 350-365.

Herzberg, G., 1950. Molecular Spectra and Molecular Structure. I. Spectra of Diatomic Molecules. Reinhold, New York, pp. 218, 240-245, and 279.

Hill, T.W., 2001. The jovian aurora oval. J. Geophys. Res. 106, 8101-8107.

Jonin, C., Liu, X., Ajello, J., James, G.K., Abgrall, H., 2000. Highresolution emission spectrum of $\mathrm{H}_{2}$. I. Cross-sections and emission yields 900-1200 Å. Astrophys. J. Suppl. 129, 247-266.

Ingersoll, A.P., Vasavada, A.R., Little, B., Anger, C.D., Bolton, S.J., Alexander, C., Klaasen, K.P., Tobiska, W.K., the Galileo SSI Team, 1998. Imaging Jupiter's aurora at visible wavelengths. Icarus 135, 251264.

Liu, X., Ahmed, S.M., Multari, R.A., James, G.K., Ajello, J.M., 1995. Highresolution electron-impact study of the far ultraviolet emission spectrum of molecular hydrogen. Astrophys. J. Suppl. 101, 375-399.

Liu, X., Shemansky, D., Ahmed, S.Y., James, G.K., Ajello, J., 1998. Electron impact excitation and emission cross-sections of the $\mathrm{H}_{2}$ Lyman and Werner systems. J. Geophys. Res. 103, 26739-26758.

Liu, X., Shemansky, D., Abgrall, H., Roueff, E., Dziczek, D., Hansen, D., Ajello, J., 2002. Time-resolved electron impact study of excitation of $\mathrm{H}_{2}$ singlet-gerade states from cascade emission in the vacuum ultraviolet region. Astrophys. J. Suppl. 138, 229-245.

Liu, X., Shemansky, D.E., Ahmed, S., Ajello, J., Abgrall, H., Roueff, E., 2003. Resonance excitation of a single rovibrational level of $\mathrm{H}_{2} \mathrm{~B}^{1} \Sigma_{\mathrm{u}}^{+}$ state and the effective excitation function of the $\mathrm{EF}^{1} \Sigma_{\mathrm{g}}^{+}-\mathrm{X}^{1} \Sigma_{\mathrm{g}}^{+}$band system. J. Phys. B At. Mol. Phys. 36, 173-196.

Livengood, T.A., Strobel, D.F., Moos, H.W., 1990. Long-term study of longitudinal dependence in primary particle precipitation in the north jovian aurora. J. Geophys. Res. 95, 10375-10388.

Mauk, B.H., Anderson, B.J., Thorne, R.M., 2003. Magnetosphereionosphere coupling at Earth, Jupiter, and beyond. In: Mendillo, M.
Nagy, A., Waite, J.H. (Eds.), Atmospheres in the Solar System: Comparative Aeronomy. In: Monogr. Ser., vol. 130. AGU, Washington, DC

Perry, J.J., Kim, Y.H., Fox, J.L., Porter, H.S., 1999. Chemistry of the jovian auroral ionosphere. J. Geophys. Res. 104, 16541-16565.

Prangé, R., Rego, D., Pallier, L., Connerny, J.E., Zarka, P., Queinnec, J., 1998. Detailed study of FUV jovian auroral features with the postCOSTAR HST faint object camera. J. Geophys. Res. 103, 2019520215.

Pryor, W.R., Stewart, A.I., Simmons, K.E., Ajello, J.M., Tobiska, W.K., Clarke, J.T., Gladstone, G.R., 2001. Detection of rapidly varying $\mathrm{H}_{2}$ emissions in Jupiter's aurora from the Galileo orbiter. Icarus 151, 314 317

Pryor, W.R., 22 colleagues, 2005. Cassini UVIS observations of Jupiter's auroral variability. Icarus $78,312-326$.

Shemansky, D.E., Ajello, J.M., 1983. The Saturn spectrum in the EUVelectron excited hydrogen. J. Geophys. Res. 88, 459-464.

Vasavada, A.R., Bouchez, A.H., Ingersoll, A.P., Little, B., Anger, C.D., 1999. Jupiter's visible aurora and Io footprint. J. Geophys. Res. 104, 27133-27142.

Waite, J.H., Cravens, T.E., Kozyra, J., Nagy, A.F., Atreya, S.K., 1983. Electron precipitation and related aeronomy of the jovian thermosphere and ionosphere. J. Geophys. Res. 88, 6143-6163.

Wolven, B.C., Feldman, P.D., 1998. Self-absorption by vibrationally excited $\mathrm{H}_{2}$ in the Astro-2 Hopkins Ultraviolet Telescope spectrum of the jovian aurora. Geophys. Res. Lett. 25, 1537-1540.

Wong, A.S., Lee, A.Y., Yung, Y.L., Ajello, J.M., 2000. Jupiter: Aerosol chemistry in the polar atmosphere. Astrophys. J. 534, L215-L217.

Yung, Y.L., Gladstone, G.R., Chang, K.M., Ajello, J.M., Srivastava, S.K., 1982. $\mathrm{H}_{2}$ fluorescence spectrum from 1200 to $1700 \AA$ by electron impact: Laboratory study and application to jovian aurora. Astrophys. J. 254, L65-L69. 